\title{
Time-dependence Effects in Photospheric-Phase Type II Supernova Spectra
}

\author{
Luc Dessart $^{1 \star}$ and D. John Hillier ${ }^{2}$ \\ ${ }^{1}$ Department of Astronomy and Steward Observatory, The University of Arizona, Tucson, AZ 85721 \\ ${ }^{2}$ Department of Physics and Astronomy, University of Pittsburgh
}

Accepted . Received

\begin{abstract}
Spectroscopic modeling of Type II supernovae ( $\mathrm{SNe}$ ) generally assumes steady-state. Following the recent suggestion of Utrobin \& Chugai, but using the 1D non-LTE line-blanketed model atmosphere code CMFGEN, we investigate the effects of including time-dependent terms, which are generally neglected, that appear in the statistical and radiative equilibrium equations. We base our discussion on the ejecta properties and the spectroscopic signatures obtained from time-dependent simulations, investigating different ejecta configurations (slow, standard, and fast), and covering their evolution from one day to six weeks after shock breakout. Compared to equivalent steady-state models, our time-dependent models produce SN ejecta that are systematically over-ionized, affecting helium at one week after explosion, but ultimately affecting all ions after a few weeks. While the continuum remains essentially unchanged, time-dependence effects on observed spectral lines are large. At the recombination epoch, HI lines and NaI D are considerably stronger and broader than in equivalent steadystate models, while CaII $8500 \AA$ is weakened. If time dependence is allowed for, the HeI lines at $5875 \AA$ and $10830 \AA$ appear $\sim 3$ times stronger at one week, and HeI $10830 \AA$ persists as a blue-shifted absorption feature even at 6 weeks after explosion. Time dependence operates through the energy gain from changes in ionization and excitation, and, perhaps more universally across SN types, from the competition between recombination and expansion, which in-turn, can be affected by optical-depth effects. Our time-dependent models compare well with observations of the low-luminosity low-velocity SN 1999br and the more standard SN $1999 \mathrm{em}$, reproducing the $\mathrm{H} \alpha$ line strength at the recombination epoch, and without the need for setting unphysical requirements on the magnitude of nickel mixing.
\end{abstract}

Key words: radiative transfer - stars: atmospheres - stars: supernovae: individual: 1999br, $1999 \mathrm{em}$

\section{INTRODUCTION}

Because of radiative cooling and the fast expansion of the exploding mantle of the progenitor massive star, photospheric-phase Type II SN spectra evolve rapidly. At shock breakout the spectral energy distribution (SED) peaks in the far-UV and X-rays. Subsequently the SED centers in the UV, then in the optical, and later in the infrared until the object fades from view. From a radiative transfer perspective, the cooling induces a recombination to lower ionization stages that impose a strong blanketing effect on the energy distribution. A few days after explosion, the ejecta radiate a UV-dominated SED, with HeII $4686 \AA$ (only observed at a few days after shock breakout; Dessart et al. 2007), Balmer/Paschen lines of Hydrogen, HeI $5875 \AA$, HeI $10830 \AA$, some multiplets of OII and

\footnotetext{
^ E-mail: luc@as.arizona.edu
}

NII multiplets at $4600 \AA$ (see Dessart \& Hillier 2006a; Baron et al. 2007), NII 5400 $\AA$, and a few isolated resonance lines such as MgII 2802 $\AA$ and AlIII $1859 \AA$. As the ejecta continue to cool, hydrogen eventually recombines, with a contemporaneous enhancement in line-blanketing due to the switch from FeIII to FeII. In Dessart \& Hillier (2006) and Dessart et al. (2007), we successfully modeled these epochs for SNe 1999em, 2005cs, and 2006bp, using steady-state non-LTE CMFGEN models and a power-law density distribution with an exponent of ten (the immediate postbreakout phase requires higher values for this exponent). These spectroscopic analyses deliberately focused on the first $\sim 45$ days after shock breakout, since, beyond that time, we encountered severe difficulties in reproducing the hydrogen lines. Specifically, at late times, synthetic line profiles were sizably narrower than observed, suggesting that line formation regions predicted by CMFGEN were confined to velocities/radii that were too small. Despite 
a continued success with reproducing the overall continuum energy distribution, CMFGEN failed to reproduce important line profiles, whenever hydrogen started recombining at and above the photosphere.

The $\mathrm{H} \alpha$ problem has not been clearly emphasized in the literature, where one can see a great disparity in model atmosphere assumptions and agreement between theoretical predictions and observations, but no direct link to physical/numerical issues. In the eighties and early nineties, the recognized importance of non-LTE effects confronted the strong limitations of computer technology, so that only a few species were treated in non-LTE (i.e., usually hydrogen and helium), while the metals responsible for line blanketing were treated in LTE. Eastman \& Kirshner (1989) followed this approach to model the first ten days of SN 1987A, and did not encounter obvious difficulties with hydrogen lines. Schmutz et al. (1990), using an approximate non-LTE technique, had, on the contrary, great difficulty reproducing any of the Balmer lines, suggesting clumping as the culprit. Höflich (1988) reproduced the SN 1987A spectral evolution and the hydrogen lines, over many months, using a large "turbulent" velocity. In the more sophisticated non-LTE CMFGEN (Hillier \& Miller 1998; Dessart \& Hillier 2005a) models presented in Dessart \& Hillier (2006a), we found that decreasing the turbulent velocity weakened line-blanketing effects and increased, although only modestly, the strength of hydrogen lines. However, beyond 40 days after explosion, this tuning had no longer any important influence on the hydrogen lines.

The non-LTE model atmosphere code PHOENIX predicts strong Balmer lines at the hydrogen recombination epoch (Mitchell et al. 2001; Baron et al. 2003), but this may stem from their adoption of non-thermal ionization/excitation due to ${ }^{56} \mathrm{Ni}$ at the photosphere, sometimes just a few days after explosion and in mass shells moving at $\geqslant 10000 \mathrm{~km} \mathrm{~s}^{-1}$ in SN 1987A (Mitchell et al. 2001) or $\sim 9000 \mathrm{~km} \mathrm{~s}^{-1}$ in SN 1993W (Baron et al. 2003). Hydrodynamical simulations of core-collapse $\mathrm{SNe}$ predict that ${ }^{56} \mathrm{Ni}$ has velocities of at most $\sim 4000 \mathrm{~km} \mathrm{~s}^{-1}$, the nickel fingers being strongly decelerated at the H/He interface (Fryxell et al. 1991; Kifonidis et al. 2000, 2003). The magnitude of this disagreement extends far beyond the uncertainties of explosion models and suggests a genuine incompatibility.

Interestingly, Hi lines remain strong for months in all Type II SNe, in objects as diverse as the "peculiar" SN 1987A, the "plateau" SN 1999em, and the "low-luminosity" SN 1999br. CMFGEN models computed for these objects were unable to reproduce Balmer lines after $\sim 4$ days in SN 1987A, 40 days in SN 1999em, and $\sim 20$ days in SN 1999br, all coincident with hydrogen recombination in the ejecta. These three SNe have very different inferred ejecta properties and observed light curves. SN 1999br even synthesized an order of magnitude less ${ }^{56} \mathrm{Ni}$ than average (Pastorello et al. 2004) for the Type II class. The only common property between these objects, which is connected to the $\mathrm{H} \alpha$ problem, is the recombination of the ejecta to a lower ionization state at the corresponding epoch.

Recently, Utrobin \& Chugai (2005, UC05) proposed that the effect of time-dependence, and the energy associated with changes in ionization/excitation, lead to a strong $\mathrm{H} \alpha$ line profile in $\mathrm{SN}$ 1987A during the recombination epoch. They also found that barium lines were affected, and that, with their more consistent approach, BaII $6142 \AA$ could be fitted using the LMC metallicity value. The steady-state models of Mazzali et al. (1992) supported instead an abundance enhancement of five.

Time dependence has been invoked in the past by
Fransson \& Kozma (1993) to explain the late-time light curve of SN 1987A, and the theoretical study of Pinto \& Eastman (2000a. b) showed that time dependence in the radiation field had a critical impact on the radiative transfer in Type Ias. Pinto \& Eastman (2000a b), however, treat the material in LTE, i.e., do not solve the rate equations, focusing instead on the time-dependent diffusion of photons through an optically thick Type Ia SN ejecta. Similarly, Kasen et al. (2006) neglect explicit time dependence in the rate equations, computing the ionization and excitation state of the medium in LTE. Thus, although there is at present growing interest in accounting for time dependence in the radiation field, the oftenused expedient of LTE, to maintain low CPU costs, has forced the neglect of both non-LTE and time-dependence in the level populations. One exception to these time-dependent LTE approaches is the work of Höflich (2003), who treats time-dependence in the rate equations but, to our knowledge, has not discussed the associated effects on Type II SN spectra. In the present study, we investigate thoroughly the effects of time-dependence in the rate equations and their impact on inferred ejecta properties. Note that time dependence in the radiation field is accounted for by adjusting the base luminosity so that the emergent synthetic flux matches the observed flux, using the bolometric-light evolution of SN 1999em as a guide (Dessart \& Hillier 2006a).

Here, we report the salient features of several time-dependent non-LTE CMFGEN simulations, covering the early evolution for a range of Type II SN ejecta. We confirm the results of UC05 that time-dependence induces an over-ionization of the recombining ejecta and that it solves the $\mathrm{H} \alpha$ problem. However unlike UC05, we self-consistently solve the radiation transfer equation - the coupling between the level populations and the radiation field is calculated and fully allowed for. Our more comprehensive study of the full spectrum further predicts that all lines, not just those of hydrogen, are significantly affected. The ionization of the ejecta and its evolution are so strongly modified that we predict even HeI $10830 \AA$ many weeks after explosion.

In the next section, we present out treatment of timedependence in CMFGEN, which focuses here on the terms appearing in the statistical and radiative equilibrium equations (an appendix also provides further details). In $\S 2.5$, we present the various model calculations performed to illustrate our discussion. In $\S 3$, we present our results, discussing the effects of time dependence on the ejecta properties as well as the associated spectroscopic signatures. We then present in $\S 4$ a comparison with a few representative observations, focusing on the well observed Type II Plateau SN 1999em and the low luminosity Type II SN $1999 \mathrm{br}$. In $\S 5$, we discuss the implications of such time-dependent effects on our understanding and on our modeling of photospheric-phase Type II SN spectra, before giving our conclusions in $\S 6$. Note that the salient features and key results presented here are also available in a concise, "letter", format in Dessart \& Hillier (2006b), to which we refer the hurried reader.

\section{TREATMENT OF TIME DEPENDENCE}

\subsection{Statistical Equilibrium Equations}

The general form of the time dependent statistical equilibrium equation is

$\frac{\partial n_{i}}{\partial t}+\nabla \cdot\left(n_{i} v\right)=\sum_{j}\left(n_{j} R_{j i}-n_{i} R_{i j}\right)$ 
where $v$ is the velocity, $n_{i}$ is the population of state $i$, and $R_{i j}$ is the transition rate from state $i$ to state $j$. The forms of $R_{i j}$ and $R_{j i}$ are standard, and can be found, for example, in Mihalas (1978). For simplicity we have not distinguished between the different processes (collisional, bound-free, and bound-bound) entering these rates.

In massive stars, the winds can be approximated as stationary. In such cases we consider a Eulerian formulation, and take $\partial n / \partial t$ to be zero. The $\nabla .\left(n_{i} v\right)$ term is the advection term, and is usually neglected, however it can become important in the outer regions of the wind, since the ratio of the recombination time, $1 / \alpha n_{e}$, to the flow time, $r / v$, scales as the radius, $r$ (here, $\alpha$ is the recombination coefficient).

Supernovae are obviously time dependent, and in this case it makes sense to use a Lagrangean formalism. Using the continuity equation

$\frac{D \rho}{D t}+\rho(\nabla \cdot v)=0$,

the statistical equilibrium equations become

$\rho \frac{D n_{i} / \rho}{D t}=\sum_{j}\left(n_{j} R_{j i}-n_{i} R_{i j}\right)$,

where $D / D t$ is the comoving derivative. For a Hubble flow,

$\frac{1}{r^{3}} \frac{D r^{3} n_{i}}{D t}=\sum_{j}\left(n_{j} R_{j i}-n_{i} R_{i j}\right)$,

which is the form we used in these calculations.

To solve these equations we use implicit first order differencing in the Lagrangean frame. Thus the equations to be solved become

$\frac{\left(n_{i}\right)_{k}-\left(n_{i}\right)_{k-1}\left(r_{k-1} / r_{k}\right)^{3}}{\Delta t}=\sum_{j}\left(n_{j} R_{j i}-n_{i} R_{i j}\right)_{k}$

where $k-1, k$ refer to values at consecutive time steps but with the same comoving coordinate. For a Hubble flow, we can simply use the velocity as the Lagrangean co-ordinate. The solution of these equations proceeds in an identical fashion to that used to solve the steady-state statistical equilibrium equations (Hillier 1987; Hillier 1990; Hillier \& Miller 1998); the only distinction is that we have a "source" term that comes from the populations at an earlier time step.

\subsection{Energy balance}

The energy equation is

$\rho \frac{D e}{D t}-\frac{P}{\rho} \frac{D \rho}{D t}=4 \pi \int\left(\chi_{\nu} J_{\nu}-\eta_{\nu}\right)$,

where $e$ is the internal energy/unit mass, $\chi_{\nu}$ the opacity, $\eta_{\nu}$ the emissivity, and $J_{\nu}$ the mean intensity, and we have ignored other forms of energy deposition (such as nuclear decay). $e$ can be written in the form

$e=e_{K}+e_{I}$

where

$e_{K}=\frac{3 k T\left(n+n_{e}\right)}{2 \mu m n}$,

and
$e_{I}=\sum_{i} \frac{n_{i} E_{i}}{\mu m n}$

In the above, $n$ is the total particle density (excluding electrons), $n_{e}$ is the electron density, and $E_{i}$ is the total energy (excitation and ionization) of state $i$. This equation is solved implicitly, via linearization, and is no more difficult to treat than the regular constraint of radiative equilibrium.

\subsection{Are the time-dependent terms important?}

Below we examine when the time dependent terms in the statistical equilibrium equations will play an important role in determining the radiative transfer in $\mathrm{SN}$ envelopes. To do this, we need to compare the time it takes to replenish the population of individual levels with the flow time. As noted previously, the flow-time scale is simply $r / v$, and for a Hubble flow this is texp, where texp is the time since the explosion.

We will only examine the ionization equilibrium in detail, since it is the ionization equilibrium that will be most affected by the time-dependent terms - statistical equilibrium of excited levels will generally be satisfied because the radiatives rates tend to be large $\left(A>10^{6} \mathrm{~s}^{-1}\right)$, and the transitions have low (or moderate) optical depths.

The simplest recombination timescale can be found by estimating the time it takes, at fixed electron density, for all ions to recombine. Thus the recombination timescale for hydrogen, $t_{\text {rec }}$ is simply given by

$t_{\mathrm{rec}}=\frac{n\left(\mathrm{H}^{+}\right)}{n\left(\mathrm{H}^{+}\right) n_{e} \alpha}=\frac{1}{n_{e} \alpha}$.

Using $\alpha=\alpha_{B}=2.59 \times 10^{-13} \mathrm{~cm}^{3} \mathrm{~s}^{-1}$ at $10,000 \mathrm{~K}$ (Osterbrock 1974), gives

$t_{\text {rec }}=\frac{4.47 \times 10^{7}}{n_{e}}$ days.

We use $\alpha_{B}$ since in SN conditions every direct recombination to the ground state will generally be followed by an ionization. The recombination time scale for HeI is very similar to that of HI. Since the comoving density is $\propto 1 / t^{3}$, the ratio of recombination time to the flow time, at a given velocity, scales as $t^{2}$. Thus the ionization must eventually become frozen, and first at high velocities.

The previous discussion refers to the case when we can ignore optical depth effects and the existence of metastable states. When optical depth effects are important, and/or there exist low lying metastable states, it is more difficult to ascertain the relevant recombination time scale. In such cases, it is the net flow of electrons to the ground state that will set the ionization equilibrium.

As an illustration consider a two level atom with continuum. The relevant equations are

$\frac{D n_{1}}{D t}=n_{2} R_{21}-n_{1} R_{12}$

and

$\frac{D n_{2}}{D t}=n_{e} n_{I} \alpha-n_{2} P_{2 I}+n_{1} R_{12}-n_{2} R_{21}$,

and

$n_{1}+n_{2}+n_{I}=n$.

Here $n_{I}$ is used to denote the ion population, $P_{2 I}$ is the photoionization rate, $R_{21}$ refers to the combined rate for processes which couple level 2 to 1 (bound-bound, collisional, two photon decay), 
while $R_{12}$ refers to processes that excite an electron from level 1 to 2 . We assume that the ground state continuum is in detailed balance. For a strict two-level atom $\alpha=\alpha_{I 2}$ but for these discussions we can take $\alpha=\alpha_{B}$.

In the absence of the time-dependent terms, the ground state equation reduces to

$n_{2} R_{21}=n_{1} R_{12}$.

In the case of HeI the main decay route from the $2 \mathrm{~s}^{1} S$ state is two photon decay (with $A=52 \mathrm{~s}^{-1}$ ), while collisional excitation might be the main reverse mechanism.

When we include the time-dependent terms, the equation for level 1 can reduce to

$\frac{D n_{1}}{D t}=n_{2} R_{21}$

and that for level 2 becomes

$n_{2} P_{2 I}=n_{e} n_{I} \alpha$,

since the decay term, for a metastable level or for a level decaying through an optically thick transition, can be small. The effective recombination time scale, $t_{\text {eff }}$, is (since $D n_{1} / D t=-D X^{+} / D t$ ) thus

$$
\begin{aligned}
& t_{\text {eff }}=\frac{n_{I}}{D n_{1} / D t} \\
& =\frac{n_{I}}{n_{2} R_{21}} \\
& =\frac{n_{I}}{n_{e} n_{I} \alpha R_{21} / P_{2 I}} \\
& =\quad \operatorname{trec} \frac{P_{2 I}}{R_{21}} \text {. }
\end{aligned}
$$

Thus, if the photoionization rate $P_{21}$ is much greater than the decay rate $R_{21}$, the effective recombination time scale will be considerably longer than the classic recombination time scale.

The expression for $P_{2 I}$ is

$P_{2 I}=\int_{\nu_{o}}^{\infty} \sigma\left(\frac{4 \pi}{h \nu}\right) J_{\nu} d \nu$

where $\sigma$ is the photoionization cross-section and $h$ is Planck's constant. Assuming $J_{\nu}=W B_{\nu}$ (a diluted blackbody), $\sigma=$ $\sigma_{o}\left(\nu_{o} / \nu\right)^{2}$, and $h \nu_{o} / k T>>1$, we have

$$
\begin{aligned}
P_{2 I}= & 5.8 \times 10^{6} W\left(\frac{\sigma_{o}}{10^{-18}}\right)\left(\frac{\nu_{o}}{10^{15} \mathrm{~Hz}}\right)^{2} \\
& \left(\frac{T}{10^{4} \mathrm{~K}}\right) \exp \left(-h \nu_{o} / k T\right) \mathrm{s}^{-1}
\end{aligned}
$$

For He I $2 \mathrm{~s}^{1} S$, we have $A($ two phot $)=52 \mathrm{~s}^{-1}, \nu_{o}=0.960 \times$ $10^{15} \mathrm{~Hz}, \sigma_{o}=8.90 \times 10^{-18} \mathrm{~cm}^{2}$, and, assuming $W=1$, and $T=10^{4} \mathrm{~K}$, we find

$$
t_{\text {eff }}=9.2 \times 10^{3} t_{\text {rec }} .
$$

Thus, it is apparent that the effective recombination time scale is much longer than the classic recombination time scale, and could easily be comparable to the flow time scale.

We appreciate that the true situation is more complex than that discussed above. For HeI the $2 \mathrm{~s}^{1} S$ and $2 \mathrm{p}^{3} S$ state are both metastable, and there is a collisonal coupling between the singlet and triplet terms. The $2 \mathrm{~s}^{1} S$ state can decay directly to the ground state, for example, by two-photon decay, by collisional deexcitation, and by collisional excitation to the $2 \mathrm{p}^{1} \mathrm{P}^{\mathrm{o}}$ state followed by a radiative decay. Further, the effective recombination rate will substantially exceed the classic recombination time scale only if the resonance line is optically thick. Despite these caveats, it is apparent that the time-dependent terms might be important even at the photosphere, and must be included. A simple comparison between the flow time scale and the classic recombination time scale can greatly underestimate the importance of these time-dependent terms.

In the Appendix we examine, in detail, the various processes that influence the ionization of helium at a representative depth, and in a typical model, and where the flow time is significantly greater than the classic recombination time. In that appendix we show that the combination of optical depth effects, and the existence of metastable levels, leads the time-dependent terms to cause a delay in the recombination of $\mathrm{He}^{+}$to neutral $\mathrm{He}$.

For $\mathrm{HI}$, the $2 \mathrm{~s}$ state is metastable, but in atmospheric calculations (as is done here) it is usually assumed to be in LTE with respect to the $2 p$ state (i.e., the $2 s$ and $2 p$ states have the same departure coefficients). Thus the relevant time scale is set by radiative decay from level $2 p$ to 1 s, and, hence, by the Ly $\alpha$ optical depth, as was previously noted by UC05. Other processes or configurations (e.g., overlapping lines, continuum opacity) can also be important since they can destroy a Ly $\alpha$ photon, facilitating a "real" recombination to the ground state.

Finally we stress, as apparent from the above discussion, that the time-dependent term does not directly change the ionization equilibrium (unlike the situation at low electron densities). Rather, it prevents the decay of electrons from the $n=2$ states (for $\mathrm{H}$ I and $\mathrm{He}$ I) to the ground state. The ionization of H I and He I can then be maintained by ionizations from these excited states.

\subsection{Radiative Transfer}

Explicit in our calculations is the assumption that we can solve for the level populations and temperature structure neglecting the relativistic and the time dependent terms in the radiative transfer equation. While it is moderately easy to include the relativistic terms (e.g., Hauschildt 1992) the time dependent term is equally important and must be included for consistency (Pinto \& Eastman $2000 \mathrm{a}$ b). In hydrodynamic models, both terms are routinely (and must be) included, both for the hydrodynamics and computing the light curve.

However for spectral calculations it is usual to follow a stellar approach and assume that we can compute a static atmosphere by specifying the luminosity via the diffusion approximation at the lower boundary. Studies of the importance of the relativistic terms have been done, for example, by Hauschildt et al. (1991), or by Jeffery (1993), and shown to be important. What is not clear, however, is what effect the terms will have on the distances based on the Expanding Photosphere Method (Dessart \& Hillier 2005b), something that we are currently investigating. Very preliminary work suggests that the inclusion of relativistic terms, while producing spectra that are very similar, could lead to changes in EPM distance by up to $10 \%$. However, we do not expect that the inclusion of these terms will effect the conclusions in this paper; the photospheric temperatures are similar and the importance of the time-dependent terms arises primarily through atomic physics, and should not be sensitive to radiative transfer affects.

\subsection{Presentation of models}

We adopt a different procedure to model $\mathrm{SN}$ spectra from that employed previously in Dessart \& Hillier (2005a. b, 2006a) by modeling the time evolution of a larger portion of the ejecta, and in a 
Table 1. CMFGEN ejecta characteristics. From left to right, the column refers to the model name, the initial base radius $R_{0}$, the base velocity $v_{0}$, the initial base density $\rho_{0}$, the total mass of the ejecta covered by the CMFGEN grid $M_{\text {ejecta }}$, the initial and final times $t_{i}$ and $t_{f}$, the initial and final base electron-scattering optical depths $\tau_{\mathrm{es}, \mathrm{i}}$ and $\tau_{\mathrm{es}, \mathrm{f}}$, assuming full and uniform ionization ( $\kappa_{\mathrm{es}}=0.30$ ), the initial and final base electron-scattering optical depths $\tau_{\mathrm{es}, \mathrm{i}}$ and $\tau_{\mathrm{es}, \mathrm{f}}$ computed with CMFGEN including time-dependent terms in the statistical and radiative equilibrium equations, and, in the last column, the final base electronscattering optical depth computed with CMFGEN under the assumption of steady-state. Note how the value of $\tau_{\mathrm{es}, \mathrm{f}}$ diminishes from the assumption of fixed and full ionization, CMFGEN with time-dependence, and CMFGEN without time-dependence.

\begin{tabular}{|c|c|c|c|c|c|c|c|c|c|c|c|c|}
\hline & & $\begin{array}{c}R_{0} \\
10^{10} \mathrm{~cm}\end{array}$ & $\begin{array}{c}v_{0} \\
\mathrm{~km} \mathrm{~s}^{-1}\end{array}$ & $\begin{array}{c}\rho_{0} \\
10^{-10} \mathrm{~g} \mathrm{~cm}^{-3}\end{array}$ & $\begin{array}{c}M_{\text {ejecta }} \\
\mathrm{M}_{\odot}\end{array}$ & \multicolumn{2}{|c|}{ days } & \multicolumn{2}{|c|}{ Fixed ionization } & \multicolumn{2}{|c|}{$\begin{array}{c}\text { CMFGEN } \\
\text { D/DT }\end{array}$} & $\begin{array}{c}\tau_{\mathrm{es}, \mathrm{f}} \\
\text { CMFGEN } \\
\text { no D/DT }\end{array}$ \\
\hline Baseline & A & 25238 & 3471 & 4.2 & 6.17 & 8.42 & 48.68 & 3686 & 111.4 & 3640 & 45.0 & 0.9 \\
\hline Slow & B & 14000 & 2000 & 8.0 & 2.00 & 8.10 & 42.82 & 3886 & 143.9 & 3840 & 9.8 & 0.6 \\
\hline Fast & $\mathrm{C}$ & 25238 & 4400 & 4.2 & 6.17 & 6.63 & 35.51 & 3686 & 135.4 & 3640 & 20.7 & 0.4 \\
\hline Early & $\mathrm{D}$ & 6539 & 4350 & 4.0 & 0.10 & 1.74 & 6.76 & 904 & 60.2 & 900 & 51.9 & 51.7 \\
\hline
\end{tabular}
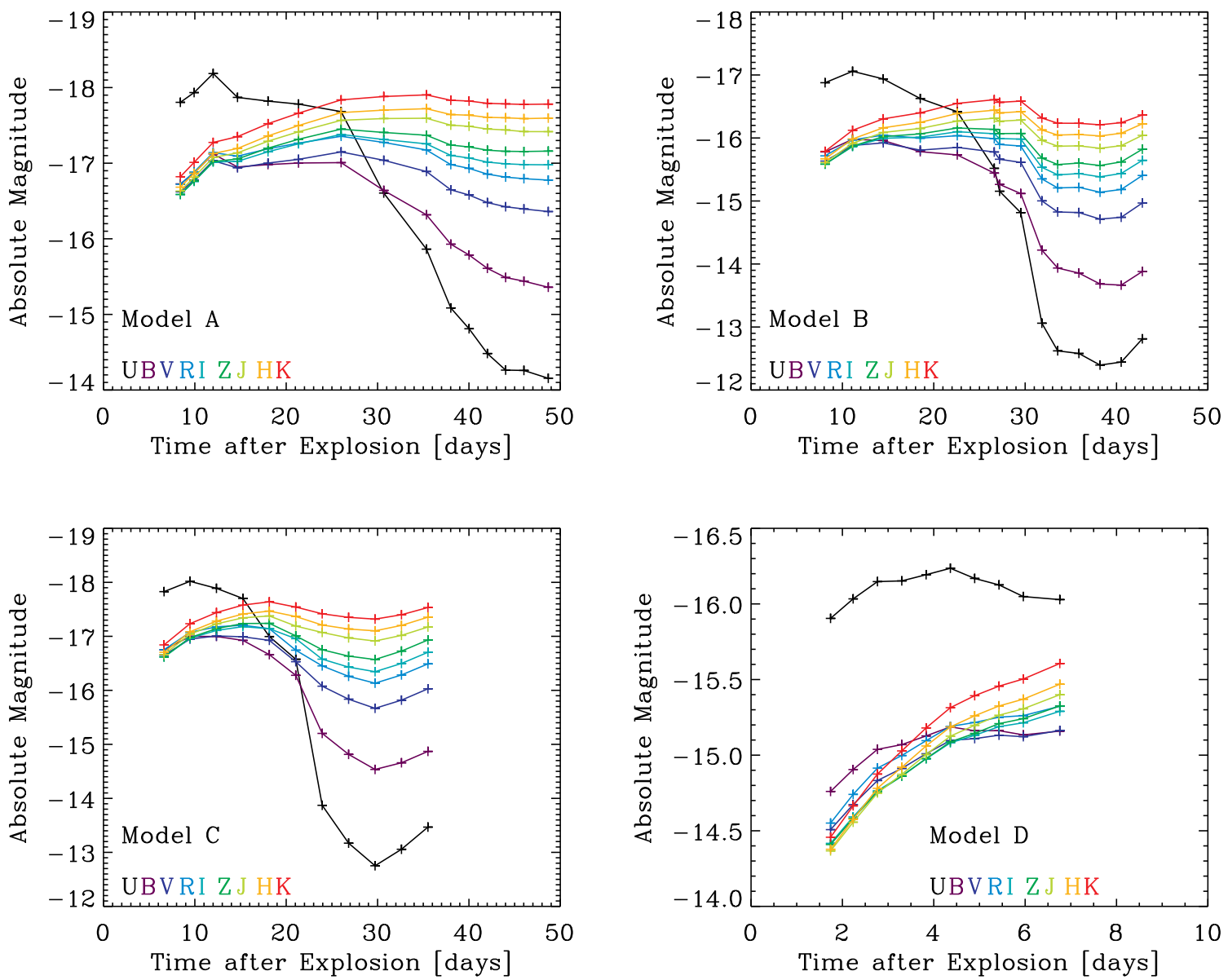

Figure 1. Synthetic light curves for the baseline model (A), the slow (B) and the fast (C) version of model A, and the "early" model (D), which covers the first week after explosion. These models correspond to standard (models A and C) and underluminous (models B and D) Type II SNe, and reproduce approximately the evolution of their bolometric luminosity and color, with a bolometric luminosity that decreases and an SED that peaks further and further to the red with time. Note that in some models, the emergent luminosity shows a small rise at the end of the sequences, resulting from a slight inadequacy in the imposed flux at the inner boundary. [See the electronic edition of the Journal for a color version of this figure.]

Lagrangean spirit. Each model is stepped in time using the implicit method outlined above. We have tested the dependence of our results on the adopted time increment. Two sequences evolved with a 1 day increment or a 2 day increment, but starting and ending with the same model input parameters, yield an SED at the final time that agree to better than $0.1 \%$ on average, departures peaking at $1 \%$ at certain UV wavelengths (coinciding with strong lines but associated with spectral regions of negligible flux). In practice, we increase the time increment (slightly) with the time elapsed since explosion, using a fraction of a day within the first week, but $\sim 3$ days at a few weeks after shock breakout. For simplicity, we choose the same minimum and maximum velocities for all models in a given 

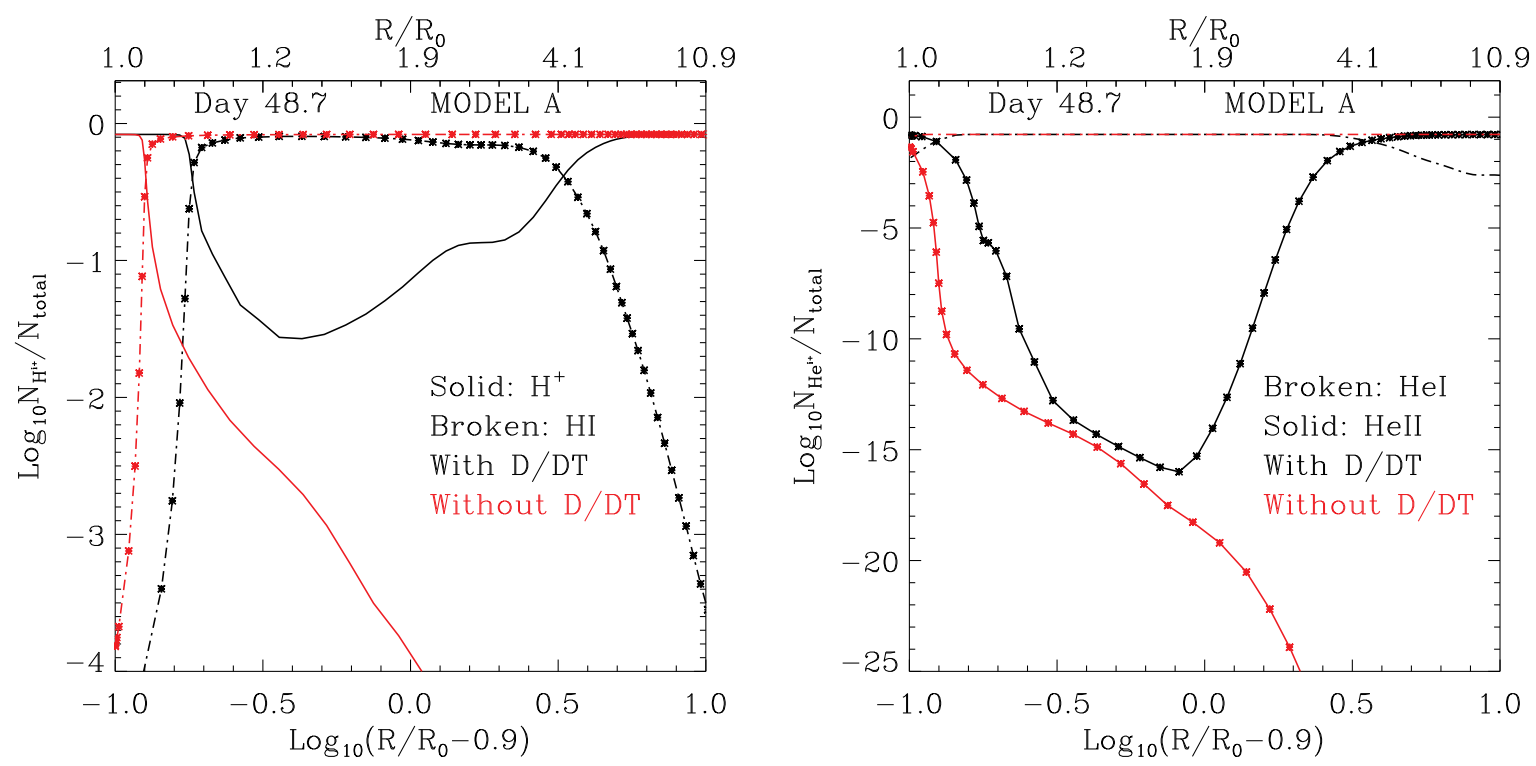

Figure 2. Left: Radial variation of the ionization fraction of Hydrogen (HıI: solid line; HI: Broken line) for the baseline model A with time dependence (black) and without (red), and at 48.7 days after explosion, but otherwise having identical parameters. Note the over-ionization of hydrogen at large radii, not present in its neutral state despite the very late time, akin to a frozen-in ionization. Symbols give the positions of the CMFGEN adaptive-grid points, for each model but for only one curve. Note the presence of the well-resolved recombination front near the base, deeper-in for the steady-state CMFGEN model. Right: Same as left, but for the ionization fraction of helium (HeII: solid line; HeI: Broken line). Notice the same frozen-in ionization at large radii. [See the electronic edition of the Journal for a color version of this figure, and the text for discussion.]

sequence. Because we neglect the relativistic and time dependent terms from the transfer equation, the base luminosity is a model parameter only, and does not reflect the true physical base luminosity. Implicit in our method is the assumption that the observed spectrum primarily depends on the local photospheric conditions as set by the observed luminosity. We use the same approach in the steady-state modeling, although in that case we only need to model the atmosphere to a depth where we can utilize the diffusion approximation, usually corresponding to a Rosseland optical depth of a few tens. Here, the initial inner radius of the computation is chosen so that it remains optically thick in the continuum over the entire time sequence, thereby ensuring at all times the adequacy of the diffusion approximation employed at this inner boundary. (Note that we refer to the photosphere as the location where the inward-integrated continuum optical depth equals two thirds.) To isolate the effects associated with time dependence, we neglect any chemical stratification (homogeneous ejecta) and assume a uniform power-law density distribution $(d \ln \rho / d \ln R=-n)$, with the exponent $n$ set to 10 .

We illustrate time-dependence effects with four different models. We set up a "baseline" model (A) that mimicks the properties of SN 1999em (Dessart \& Hillier 2006a). In practice, we initially map onto the CMFGEN grid an envelope with the following properties: $R_{0}=2.524 \times 10^{14} \mathrm{~cm}\left(R_{0}\right.$ is the base radius), $v_{0}=3470 \mathrm{~km} \mathrm{~s}^{-1}\left(v_{0}\right.$ is the base velocity), $R_{0}^{3} \rho_{0}=6.781 \times 10^{33} \mathrm{~g}$ ( $\rho_{0}$ is the base density), $\rho(R)=\rho_{0}\left(R_{0} / R\right)^{n}$ with the density exponent $n=10$ ( $R$ is the radius), and $L_{0}=3.6 \times 10^{11} \mathrm{~L}_{\odot}\left(L_{0}\right.$ is the base luminosity). The maximum radius is 16 times the value of the base radius $R_{0}$ (this choice ensures that at the outer CMFGEN boundary, the radiation field is more decoupled from matter at all frequencies and free streaming, i.e., the Eddington factor is close to unity). These initial conditions correspond to a base Rosseland optical depth of 3640 , a total mass of $6.17 \mathrm{M}_{\odot}$, and, assuming ho- mology, a time after explosion of 8.4 days. We adopt a fixed composition (i.e,. chemical homogeneity), with values consistent with the red-sugergiant progenitor of SN 1999em (Dessart \& Hillier 2006a; Smartt et al. 2002). Adopted abundances, given by number, are: $\mathrm{H} / \mathrm{He}=5, \mathrm{C} / \mathrm{He}=0.00017, \mathrm{~N} / \mathrm{He}=0.0068, \mathrm{O} / \mathrm{He}=0.0001$, and all metal species are at the solar value. We also set up a slow (B; SN 1999br-like) and a fast (C) model, differing in the velocity of its inner mass shell, i.e., $2000 \mathrm{~km} \mathrm{~s}^{-1}\left(4400 \mathrm{~km} \mathrm{~s}^{-1}\right)$ rather than $3471 \mathrm{~km} \mathrm{~s}^{-1}$. The inner radius is also adjusted so that we start in these three cases at about one week after explosion. Finally, we set up an "early-time" model (D) that starts at $\sim 2$ days after explosion. In this model, we employ a slightly different composition for $\mathrm{CNO}$ elements, with $\mathrm{C} / \mathrm{He}=0.0001, \mathrm{~N} / \mathrm{He}=0.0013, \mathrm{O} / \mathrm{He}=$ 0.0016 (Dessart et al. 2007). We give a synopsis of all these model characteristics in Table 1 Apart from the "early" model that covers only the first week, we evolve these models from one week up to about 6 weeks after shock breakout.

In our current approach, each ion-level population is evolved in a time-dependent non-LTE fashion, and thus, for simplicity, we adopt a unique model atom for all steps in a given model time sequence. For the baseline, slow, and fast models, we treat H I $(30,20)$, He I $(51,40)$, He II $(5,5)$, C II $(59,32)$, C III $(20,12)$, C IV $(14,9)$, N I $(104,44)$, N II $(41,23)$, N III $(8,8)$, O I $(75,23)$, O II (111,30), O III $(46,26)$, Na I $(71,22), \mathrm{Mg}$ II $(65,22)$, Si II $(59,31)$, Si III $(53,29)$, Ca II $(77,21)$, Ti II $(152,37)$, Fe II $(309,116)$, Fe III $(477,61)$, Fe IV $(282,50)$, and Ni II $(93,19)$, where the parenthesis contains the number of full- and super-levels (see Hillier \& Miller 1998 for details). Compared to the model atom used in Dessart \& Hillier (2006a), we omit CI, neon, aluminium, sulfur, chromium, manganese, and cobalt. Given the much higher ionization state at earlier epochs, the "early" model neglects most low-ionization species (and metals other than iron) and adds He II 

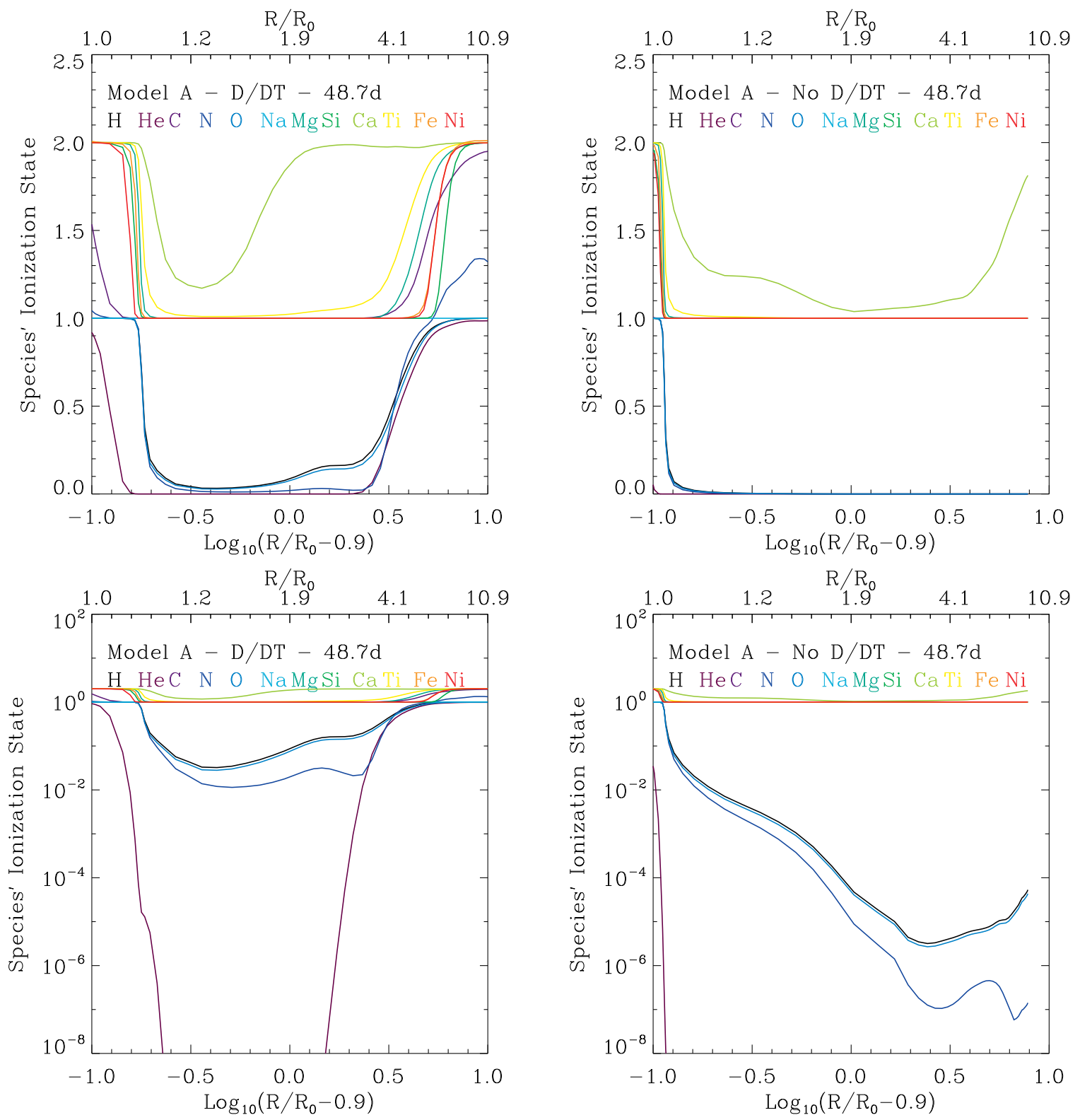

Figure 3. Top row: Comparison of the radial variation of the ionization state of all species included in model A, for the time-dependent (left) and the steadystate case (right). Note how the effects on hydrogen and helium discussed in 3.1 and illustrated in Fig. 2 apply in fact to all species, with a systematic over-ionization at large distances. Bottom row: Same as for the top-row panels, but this time shown in the log to reveal better the magnitude of the predicted difference in ejecta ionization between time-dependent and steady-state approaches. [See the electronic edition of the Journal for a color version of this figure, and the text for discussion.]

(30,13), C IV (64,59), N IV (60,34), N v (67,45), O IV (72,53), O V $(152,75), \mathrm{Fe} V(191,47)$, and Fe VI $(433,44)$.

From a converged initial steady-state model, we then step in time by $\Delta t=3$ days $(0.7$ day for the early model $\mathrm{D})$, i.e., all mass shells $m$ in the initial model are evolved from their position $R(m)$ to a new radius $R^{\prime}(m)$, with $R^{\prime}(m)=R(m)+v_{0}(m) \Delta t$, a new density $\rho^{\prime}=\rho\left(R / R^{\prime}\right)^{3}$, and the same velocity. In practice, for each new model, CMFGEN adapts its 80-point spatial grid to cover each optical-depth decade with at least five points. For model D, the grid contains only 50 radial points.

In 4 we present a sample of comparisons to observations to illustrate the performance of these time-dependent models (note that our models were not tailored to match any specific observa- tion). For the "early" model D, observations are only starting now to be available through, e.g., the SWIFT satellite (Brown et al. 2007), and the corresponding simulations for model D, thus, remain largely unconstrained (but see Quimby et al. 2007; Dessart et al. 2007). To illustrate the bolometric luminosity and color evolution of our four models, we present in Fig. 1 their light curves covering from the UV to the near-IR, with properties quite typical of Type II-P SNe (Leonard et al. 2002; Pastorello et al. 2006; Dessart et al. 2007). 


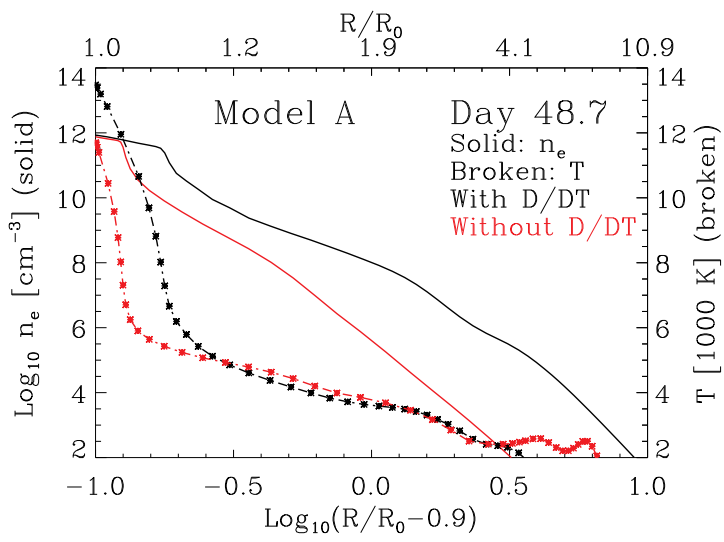

Figure 4. Radial variation of the electron density $n_{e}$ (solid line; in the logarithm, and in units of $\mathrm{cm}^{-3}$ ) and the temperature (broken line; in units of $1000 \mathrm{~K}$ ), for the time-dependent baseline model (black), and its steadystate equivalent (red). Both models have otherwise identical parameters and correspond to a time of 48.7 days after explosion. [See the electronic edition of the Journal for a color version of this figure, and the text for discussion.]

\section{RESULTS}

\subsection{Time-dependence effects on ejecta properties}

We start our discussion of time-dependence effects on Type II SNe by focusing on the properties of the ejecta. In Fig. 2 we show the ionization fraction for hydrogen (left) and helium (right) for model $\mathrm{A}$ at the end of the time sequence, 48.7 days after the explosion. Hydrogen recombination occurs just above the photosphere but less efficiently in the time-dependent model than in the steadystate model, neutral hydrogen being 3 orders of magnitude more abundant in the later model. In the time-dependent model, two additional features of interest are the presence of fully ionized hydrogen at large distances above the photosphere (the hydrogen ionization state is frozen) and the delayed recombination in the optically thick layers, at and below the photosphere (the ionization front is located further out). All these features apply to the helium ionization fraction as well, which we show in the right panel of Fig. 2 The HeII population is 15 orders of magnitude lower than that of HeI at intermediate heights, but it is comparable or stronger at large distances. Effects of time dependence can thus reach considerable levels, much beyond modulations that can be achieved through reasonable changes of (or uncertainties in) chemical abundance or ionizing fluxes.

Because metal line-blanketing plays an increasingly important role at and after the recombination epoch, time-dependence effects on metals are also of special interest. We show in Fig. 3 the ionization state of all species for model A on day 48.7. Notice how the frozen-in ionization is readily visible at large distances above the photosphere, with order of magnitude differences in ionization level between the time-dependent and steady-state approaches. Hence, the inclusion of time-dependence affects all species, irrespective, for example, of the atomic properties of the given ion, and with a magnitude that is greatest at large distances above the photosphere where the recombination timescale becomes increasingly large compared to the expansion timescale.

Finally, we show in Fig. 4 the radial variation of the temperature and the electron density for model $\mathrm{A}$ at 48.7 days after explosion. The temperature in the time-dependent model is higher in the optically-thick regions, due to the reduction of density- sensitive line emission processes that dominate radiative cooling. In the optically-thin regions, above the photosphere, the temperature is, however, quite similar between the two approaches. This result stems from the poor coupling between gas and radiation in these scattering-dominated, tenuous, ejecta. Departures from LTE are pronounced and the temperature is a secondary driver of the ejecta ionization, even in steady-state configurations.

Models B-C-D offer valuable information on the sensitivity of the above effects on the ejecta velocity (models B and C) and the epoch (model D). We find that varying the velocity of the ejecta within the range of values for slow/underluminous (model B; SN 1999br) and more standard luminosity Type II SN events (SN 1999em) has no sizeable effect on the main features of time dependence highlighted above. Recombination is inhibited, and a significant over-ionization persists far above the photosphere.

In Fig. 5. we present the mean ionic charge for the six species $(\mathrm{H}, \mathrm{He}, \mathrm{C}, \mathrm{N}, \mathrm{O}$, and Fe) included in model D (early epoch), and at 6.7 days after explosion. While hydrogen remains fully ionized, in both time-dependent and steady-state approaches, all other species show a higher ionization at large distances above the photosphere; no difference is noticeable in the vicinity and below the photosphere, contrary to model A at 48.7 days. This supports the idea that time-dependence effects operate even at early times. They do so at large distances because there, the recombination and expansion timescales are already sufficiently close, but they can also act at the photosphere because of optical depth effects and meta-stable levels (see $\$ 2.3$. It also shows that over-ionization at large distances does not stem by essence from the recombination energy (since most of that energy is stored in hydrogen and hydrogen remains fully ionized in this whole sequence) but results exclusively from the low-density fast-expansion of the SN ejecta. A corollary is that this progressive "over-ionization" does not require an extra source of ionization and excitation from, for example, ${ }^{56} \mathrm{Ni}$ (Mitchell et al. 2001; Baron et al. 2003), and operates instead, in a robust fashion, through the reduction of the effective recombination rates. Finally, this supports the idea that similar effects should apply in SN ejecta in general, since they generically possess similar low density and large velocities.

We conclude this section by presenting the evolution of the electron-scattering optical depth at the base radius $R_{0}$ of each model in Fig. 6 (see Table 1 for model parameters). Rather than having a $1 / t^{2}$ dependence (dashed lines) for an expanding ejecta with a fixed mass absorption coefficient (as in model D where the dashed and solid lines overlap; fixed composition and ionization), the curves for models A, B, and C show a change of concavity, with a slow decrease at first and a turn-over to a faster decrease after 3-6 weeks. Recombination, which is delayed at first and permits the persistence of a high free-electron density in the ejecta, does in the longer term occur and triggers a fast drop of the base electron-scattering optical depth. It remains, however, higher by up to a factor of about ten, than the value obtained in the corresponding steady-state model (for the same adopted lower bound radius in the optical-depth integral) at the ultimate time in the sequence, shown as filled colored circles. Model D, whose ejecta contains species at least once ionized throughout the sequence, has a base optical depth of 51.9 in the time-dependent approach and 51.7 in the steady-state approach, the modest difference stemming from the slight over-ionization of sub-dominant species like helium and CNO elements. (See the last columns of Table 1 for a $\log$ of these values.) 

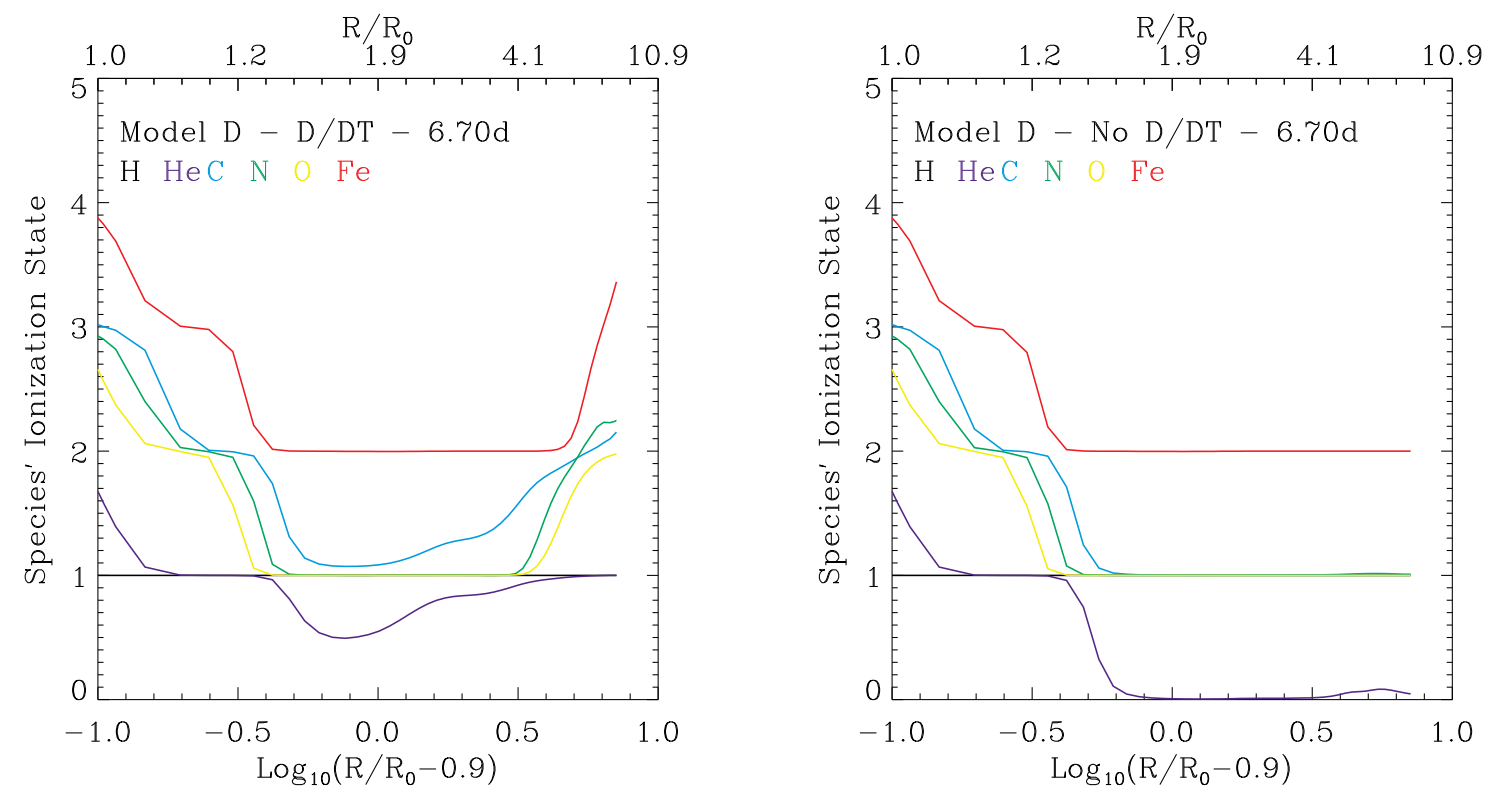

Figure 5. Comparison of the radial variation of the ionization state of all species included in model D (the model that covers the first week of evolution of a Type II SN ejecta), for the time-dependent (left) and the steady-state case (right). Note how the behaviour illustrated in Fig. 2 for hydrogen and helium is visible in fact in all species (with the exception of hydrogen which remains fully ionized in both time-dependent and steady-state approaches at such an early time; black line). Hence, even during the first week after explosion, when the ejecta density are relatively higher, time-dependence effects leave an obvious imprint on the ejecta ionization state (He, $\mathrm{C}, \mathrm{N}, \mathrm{O}, \mathrm{Fe}$ here), even though the medium, made-up primarily of fully-ionized hydrogen, retains roughly the same electron density. [See the electronic edition of the Journal for a color version of this figure, and the text for discussion.]

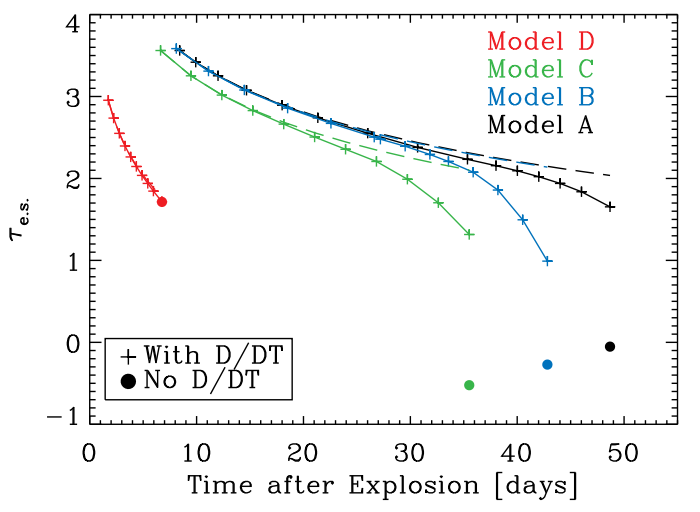

Figure 6. Time evolution of the electron-scattering optical depth $\tau_{\text {e.s. }}$. for each time-dependent model A (black; baseline model), B (blue; slow model), C (green; fast model), and D (red; early epoch model), using solid lines and cross symbols (to mark the days computed). We overplot the electron scattering optical depth for the corresponding steady-state model at the final time in each sequence (filled circle). For each model, we also draw the $1 / t^{2}$ optical-depth evolution for an ejecta with fixed electron-scattering opacity (fixed composition) and ionization (dashed line; the blue and black dashed curves overlap, and so do the red-dashed red-solid curves). [See the electronic edition of the Journal for a color version of this figure, and the text for discussion.]

\subsection{Time-dependence effects on the emergent light, from UV to near-IR, for the baseline model A}

The various investigations and analyses discussed above indicate that time-dependence effects are robust, apply to all species, even prior to the recombination epoch of hydrogen. Hence, for example, during the first week after explosion, such effects inhibit the recombination from $\mathrm{Fe}^{4+}$ to $\mathrm{Fe}^{3+}$ and $\mathrm{Fe}^{2+}$, or that of $\mathrm{He}^{+}$to $\mathrm{He}^{0+}$. Different species block light at specific wavelengths, with often a tendency to bunch-up in specific spectral regions, but with no $a$ priori correlation, and therefore we anticipate that such modulations in the ionization state of the ejecta might lead to a significant impact on the properties of the emergent light. Both the optical, dominated by $\mathrm{HI}$ and HeI lines, and the UV, dominated by metal line-blanketing due to, for example, iron, should be affected.

In Fig. 7 we present a montage of synthetic spectra showing the time evolution for the time-dependent (black) and steady-state (red) CMFGEN model A in the UV/optical (left panel), the $9000 \AA$ $-2 \mu \mathrm{m}$ region (middle panel), and the $2-5 \mu \mathrm{m}$ region (right panel) at three consecutive epochs $(8.41 \mathrm{~d}$, the start of the sequence, $30.6 \mathrm{~d}$, and the last time in the sequence at $48.6 \mathrm{~d}$ ). The SED has a peak that shifts from the UV to the optical over the 6 weeks covered, starting first as a nearly featureless continuum and then revealing more and more the effects of metal line blanketing in the UV and optical (see, e.g., Brown et al. 2007). At a qualitative level, this model sequence, which accounts for time dependence, is in agreement with what is observed in nature, for example for the Type II-P SN 2006bp (Dessart et al. 2007), and what could also be modeled with steadystate CMFGEN models. Hence, the time-dependence incorporated here has no major influence on the general morphology of the SED and its evolution with time. A corollary is that time-dependence effects on the continuum SED are weak. The effects on lines are, however, pronounced as we notice that all hydrogen lines, from the Balmer to the Paschen and to the Brackett series, are considerably weaker in the steady-state model for the last date, compared to its time-dependent equivalent, suggesting that effects do not intervene selectively, but rather that all hydrogen lines are affected.

To delineate more precisely the influence on lines, we display a montage of optical synthetic spectra for model A, correspond- 

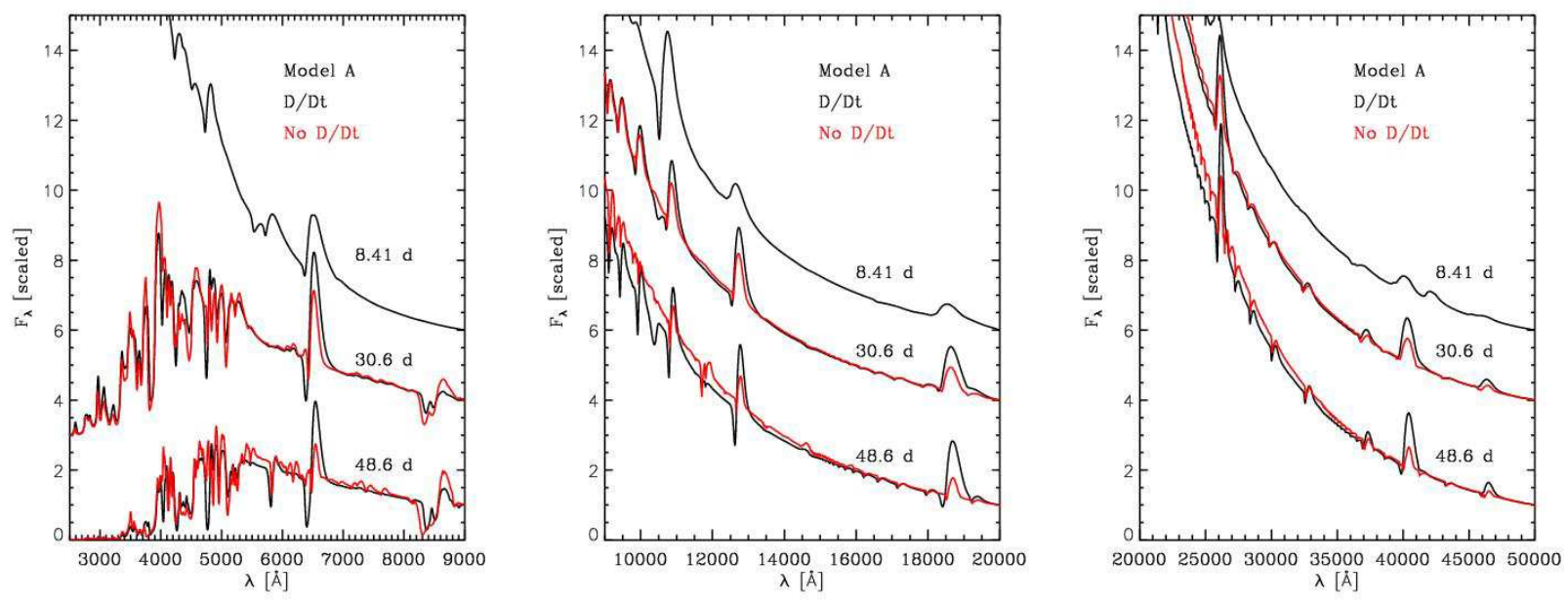

Figure 7. Montage of (scaled) synthetic spectra showing the time evolution for the time-dependent (black) and steady-state (red) CMFGEN model A in

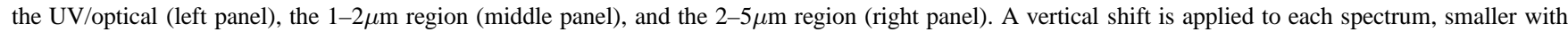
increasing time. We show the evolution for three dates, at $8.41 \mathrm{~d}$ (top curve; the time-dependent and the steady-state both start their evolution at that time and, thus, only one curve is plotted), slightly past halfway through the time sequence at $30.6 \mathrm{~d}$ (middle curve), and for the last time computed at $48.6 \mathrm{~d}$ (bottom). [See the electronic edition of the Journal for a color version of this figure, and the text for discussion.]

ing to the last time in the sequence, in Fig. 8 . In the top panel, we plot the result for the time-dependent (black) and the equivalent steady-state (red) model, while in the lower panel, we present rectified spectra obtained by post-processing the CMFGEN results and accounting for all continuum processes but only bound-bound transitions of selected ions. The contrast for Hydrogen Balmer lines is stark, and most extreme for $\mathrm{H} \alpha$, which is now as strong as observed. Helium, which does not show a single line in the steadystate model, shows a non-trivial absorption feature at $\sim 1.03 \mu \mathrm{m}$ and a very extended weak flat-topped emission profile (we will return to this line below). Continuing upwards, nitrogen and oxygen reveal only weak lines, and effects are marginal. Steady-state models show stronger absorption and weaker emission in NI lines. The same holds for MgII and SiII. In the time-dependent case, NaI $5895 \AA$ strengthens and broadens. This occurs because $\mathrm{Na}^{+}$is the dominant ionization state in both models, but the increased electron density in the time-dependent model leads to an increase in the neutral $\mathrm{Na}$ fraction. Conversely, CaII $8500 \AA$ weakens, primarily because the relative fractions of $\mathrm{Ca}^{+}$and $\mathrm{Ca}^{++}$change significantly. FeII and TiII lines are stronger in the steady state model — stronger in absorption in the UV and stronger in emission in the optical resulting from the lower ionization of the ejecta. When combined, all these effects give an SED that is most conspicuously different through the changes in the strength of $\mathrm{H} \alpha$ and CaII $8500 \AA$, whose ratio is reversed between the two approaches.

An alternative approach of strengthening the weak $\mathrm{H} \alpha$ profile, through a flattening of the density distribution 1 , leads instead, primarily, to a considerable strengthening of the CaII $8500 \AA$ multiplet, in complete contradiction with observations (Dessart \& Hillier 2005a). Time dependence offers here a means to produce a strong $\mathrm{H} \alpha$ line, while delayed recombination of calcium maintains the moderate CaII $8500 \AA$ strength. The moderate impact on metals in

1 Note that the recombination efficiency scales as the product of the electron density $n_{e}$ with the ion density $n_{i}$. Flattening the density distribution enhances the material density $\rho$ above the photosphere, but if the ejecta is recombined, the low values of $n_{e}$ and $n_{i}$ will inhibit the recombination needed to power, for example, a strong $\mathrm{H} \alpha$ line (see also 4 ]. the vicinity of the photosphere leaves metal line-blanketing only weakly affected, and it is only the strongest lines (like FeII 5169 that show both a strengthening and a broadening in the less-ionized, steady-state, model.

In Fig. 9 we plot the radial distribution of the parameter $\zeta$ of Hillier (1987) for the $\mathrm{H} \alpha$ line, a quantity that relates to the total emission in the line through the integral $\int_{R_{0}}^{R_{\mathrm{Max}}} \zeta\left(R^{\prime}\right) d \log R^{\prime}$ (or, equivalently, $\int_{V_{0}}^{V_{\operatorname{Max}}} \zeta\left(V^{\prime}\right) d \log V^{\prime}$ for homologously expanding ejecta). The curves for the time-dependent (solid line, in black apart from the final model in red) model A indicate a sustained and comparable emission distribution throughout the sequence. The peak emission shifts from $8000 \mathrm{~km} \mathrm{~s}^{-1}$ at one week to $4000 \mathrm{~km} \mathrm{~s}^{-1}$ at 6 weeks after explosion, following the expansion of the ejecta and the decrease in density. But the emission region considerably broadens with time, so that, while its inner edge recedes, its outer edge remains fairly fixed (in a Lagrangean sense). The corresponding emission region for the steady-state model (broken red line) is located deeper (the photosphere as well; see $\$ 3.1$ ) and is considerably narrower. This property translates directly in the observed $\mathrm{H} \alpha$ line properties, with a smaller line flux (weak line) confined to smaller velocity (narrow line) in the steady-state model.

To further illustrate the increase of emission-line volumes, and following Dessart \& Hillier (2005a b), we show in Fig. 10, for $\mathrm{H} \gamma$ (left-column panels), $\mathrm{H} \beta$ (middle-column panels), and $\mathrm{H} \alpha$ (rightcolumn panels), grayscale images in the $(v, p)$ plane of the flux-like quantity $p \cdot I(p)$, where $v=\left[\left(\lambda / \lambda_{0}\right)-1\right] c$ is the classical Doppler velocity, $p$ is the impact parameter in units of the photosphere radius $R_{\text {phot }}$, and $I(p)$ is the emergent specific intensity along $p$. The sum over $p$ of the quantity $p \cdot I(p)$ at $v$ corresponds to the total line flux at $v$, shown at the top of each panel (solid line). Note that for each line, we select a single HI transition to avoid the corrupting effect of line overlap as we near the Balmer jump (all other bound-bound transitions of $\mathrm{HI}$ and of other ions are ignored). A detailed interpretation of these diagrams is given in Dessart \& Hillier (2005a b) and will not be repeated here. Note the stark contrast between the considerably more extended regions of emission and absorption (in velocity or equivalently in space dimension) in the 


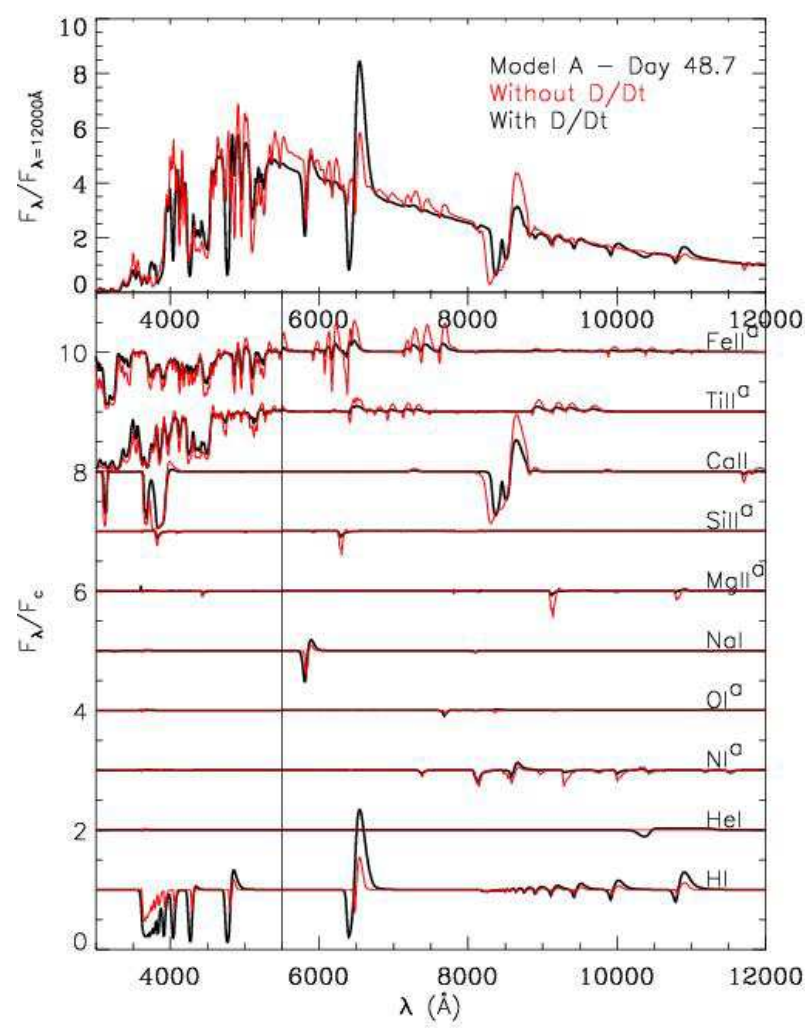

Figure 8. Top: Comparison of synthetic spectra obtained for model A and the last time in the sequence at 48.7 days, showing the results for the timedependent model (black curve) and the equivalent steady-state model on the same day (red curve). Bottom: Same as top, but for the synthetic rectified spectra (shifted vertically for visibility, and magnified by a factor of five beyond $5500 \AA$ for all species labelled with a superscript $a$ ) and allowing exclusively for bound-bound transitions of the ion species indicated on the right. We only show those species that leave an imprint on the spectrum. This figure illustrates the modest time-dependence effects over the first 6 weeks after explosion on the continuum. However, the effects on lines are significant, most noticeable on the strengthened hydrogen Balmer lines and NaI D, and the weakened CaII $8500 \AA$ multiplet lines. The enhanced recombination of the ejecta in the steady-state model results in a lower ionization state and stronger metal lines, e.g., for FeII and TilI. [See the electronic edition of the Journal for a color version of this figure, and the text for discussion.]

time-dependent models (top row) compared to the very-confined absorption and nearly nonexistant emission for the corresponding lines in the steady-state counterparts (bottom row). The same ejecta is covered by both models (same mass shells with same velocities), yet the Balmer lines show considerably broader profile in the timedependent case, mimicking an ejecta with a faster expansion rate at that given time.

The time-dependent version of CMFGEN, thus, resolves the discrepancies with hydrogen Balmer lines obtained with its steadystate version. While the difficulty with Balmer lines has been documented in the past (see, e.g., UC05), we find that time-dependence effects conspire to modify the strength of most lines, not just those of hydrogen. For example, NaI D undergoes a considerable broadening and strengthening with the treatment of time dependence, while in the past, fitting it well required enhancing the sodium abundance by a factor of about four (Dessart \& Hillier 2006a). Per-

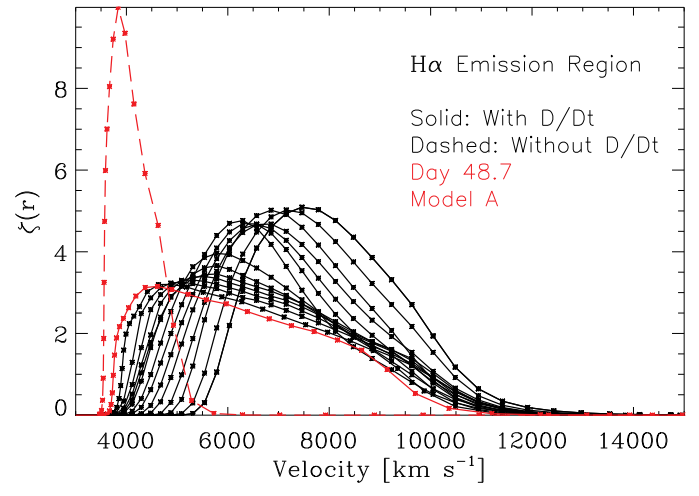

Figure 9. Solid line: Time evolution, in velocity space and for model A, of the $\xi$ parameter of Hillier (1987) for $\mathrm{H} \alpha$, which describes the spatial distribution of the corresponding line emission (the area under the curve scales with the emergent line flux). Notice how this distribution peaks at smaller radii for later times, but also the persistence of emission at large radii at all times (the last time shown in red). Broken line: Same as for the solid line but for the steady-state CMFGEN model, and only for the last time (to be compared to the solid red line). Note the deeper location of the emission, resulting in a very narrow $\mathrm{H} \alpha$ profile, in contradiction to observations at the corresponding mid-photospheric-phase epoch. [See the electronic edition of the Journal for a color version of this figure, and the text for discussion.]

haps the most extreme case in our investigation is HeI $10830 \AA$, which persists over the entire 6 weeks covered by the baseline model $\mathrm{A}$, and for which, at the last time in the sequence, we predict a non-trivial absorption strongly blueshifted from line center (at $1.038 \mu \mathrm{m}$, equivalent to $-13000 \mathrm{~km} \mathrm{~s}^{-1}$ ), and a weak and broad flattopped emissior 2 . Such a line profile morphology is synonymous with absorption/emission far above and detached from the photosphere, and usually associated with chemical stratification of the corresponding element or density kinks; here it stems solely from an ionization stratification caused by time-dependence effects. The HeI $10830 \AA$ line is entirely absent in the steady-state counterpart model three weeks after the explosion because, in the relevant region, helium atoms are once ionized with a probability of only one in $10^{20-30}$.

In Fig. 11, we reproduce Fig. 10 and illustrate the absorption/emission sites for HeI $10830 \AA$ at 48.7 days in the timedependent version of Model A. Absorption stems from the regions in the direction of the stellar disk, far from the photosphere which has a velocity of $\sim 4500 \mathrm{~km} \mathrm{~s}^{-1}$ on that day. For impact rays not intersecting the photodisk, only emission occurs, again at large distances above the photosphere, and over a small volume. The emission flux is small, appears as a flat-topped profile, and is thus barely noticeable above the continuum. Of this Her $10830 \AA$ line in the synthetic spectrum, one can only observe the strongly blueshifted absorption component, the location of the flat-topped emission overlapping with the much stronger $\mathrm{P} \gamma$ line at this late epoch. This is illustrated differently in Fig. 12 where we show

2 Chugai et al. (2007) propose that the cool dense shell that forms at the interface between the SN ejecta and the pre-SN wind is at the origin of a similar, but stronger, feature in HeI $10830 \AA$, that fits more suitably the observed line profile (Hamuy et al. 2001; Spyromilio et al. 1991). We acknowledge this possibility, and focus here only on the time-dependence effects germane to the ejecta alone. 


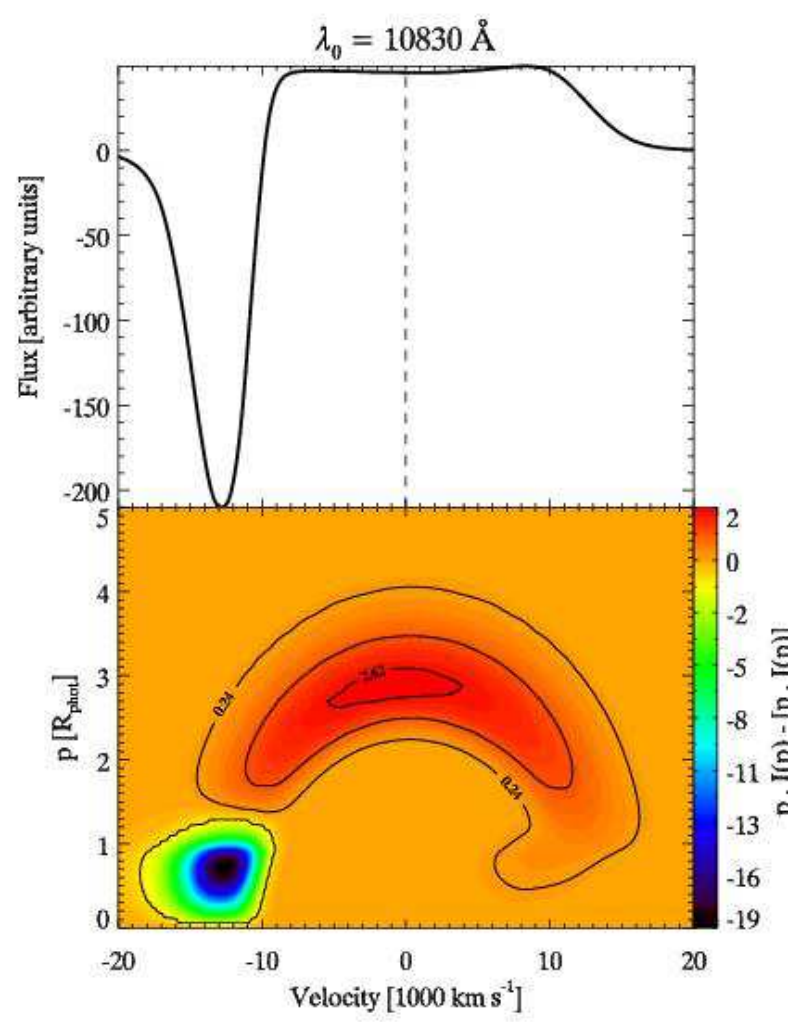

Figure 11. Same as in Fig. 10 but for the Her $10830 \AA$ line (to enhance the rendering, we subtract $p \cdot I(p)$ from the continuum-only equivalent, and add a few contours). The line profile is flat-topped, shows a "detached" blueshifted absorption, forms further out than $\mathrm{H} \alpha$ in the same timedependent model, and is completely absent in the equivalent steady-state model. [See the electronic edition of the Journal for a color version of this figure, and the text for discussion.]

a montage of synthetic spectra covering the $1-1.2 \mu \mathrm{m}$ region and the entire evolution computed for Model A. In the left panel, we show the full synthetic spectrum, i.e., including all species. In the right panel, we use the converged CMFGEN model at each time in the sequence and solve the formal solution of the radiative transfer problem by including bound-bound transitions of helium only. Note how at early times (dark curves) the profiles in the two panels match, indicating that helium is the main feature contributing in emission and absorption at early times. After three weeks, the emission is dominated by $\mathrm{P} \gamma$ and the HeI absorption gives rise to a dip, detached from the $\mathrm{P} \gamma$ absorption, and shifting further to the blue (i.e., in an opposite sense to the photosphere which recedes to smaller velocities, as well as to all other line-profile absorptions).

\subsection{Sensitivity to ejecta properties and epochs}

Besides the baseline model A discussed above, we have computed three other time sequences, as described in detail in 2.5 Model B and $\mathrm{C}$ investigate the effect of the expansion velocity of the ejecta, model $\mathrm{B}$ being also an underluminous event by design. Model D further investigates the possible (but not expected) time-dependent effects during the first week after explosion.

We show in Fig. 13 a comparison between optical synthetic spectra computed for the time-dependent (black) and the steadystate (red) Model B (left) and Model C (right), both at the last time in the sequence. Despite their different expansion velocities, models $\mathrm{B}$ and $\mathrm{C}$ show very similar time-dependence effects, with the most noticeable features being the same as for model A over the same time range: Hydrogen Balmer lines are strengthened, and so are those of the Paschen and the Brackett series (not shown here but see Fig. 7 for a guide), as well as NaI D. Here again, and by contrast, the CaII multiplet at $\sim 8500 \AA$ is weakened. Importantly, given that these models correspond to the conditions from underluminous to standard, and from slow to fast ejecta, germane to SNe $1999 \mathrm{br} / 2005 \mathrm{cs}$ and $1999 \mathrm{em} / 2006 \mathrm{bp}$, time-dependent CMFGEN models systematically predict strong spectroscopic effects. In an absolute sense, these modulations still correspond to ejecta that are fast and tenuous, where the expansion time scale is comparable to the magnitude of important rates (like that of recombination), affecting strongly the ionization equilibrium at and above the photosphere.

Turning to Model D in Fig. 14 we see that the differences, at 6.7 days, between the time-dependent and steady-state model SEDs are subtle (bottom panel). The continuum SED is essentially identical and noticeable differences are only visible in the HeI lines, particularly at $5875 \AA$ and at $10830 \AA$. Both of these are a factor of about 2-3 stronger in the time-dependent model. In the past, reproducing the strength of optical HeI lines with a typical BRG/RSG surface helium abundance has been a challenge (Eastman \& Kirshner 1989), although this difficulty disappears with a full non-LTE treatment (Dessart \& Hillier 2005a). We now find that time dependence introduces a further tuning, making optical HeI lines persist over a longer time span than is predicted by (non-LTE) steady-state models.

\section{COMPARISON WITH A SAMPLE OF OBSERVATIONS}

The motivation for the present work was to find an explanation, and a cure, for our inability with steady-state CMFGEN models to reproduce the strong $\mathrm{H} \alpha$ line at the recombination epoch. Despite all attempts, we were unable to reproduce the $\mathrm{H} \alpha$ line strength as soon as hydrogen recombination in the Type II SN ejecta takes place (Dessart \& Hillier 2006a). We reported this problem for SN $1999 \mathrm{em}$, but it is in fact a generic feature of all steady-state CMFGEN models at a few weeks after explosion. Interestingly, observations at the corresponding epoch reveal that $\mathrm{H} \alpha$ is instead the strongest line in the optical spectrum of Type II SNe, across the whole range of objects, from the peculiar SN 1987A, to the underluminous slowly-expanding SN 1999br, to the more standard SN 1999em (Type II-P). These SNe are understood to stem from core-collapse explosions of supergiant stars, and are characterized by a wide range of explosion energies (velocity distribution versus Lagrangean mass), nickel yields, and bolometric luminosity. The mechanism that can solve the present $\mathrm{H} \alpha$ discrepancy must operate at the hydrogen recombination epoch and be effective in all Type II SN ejecta.

Specifically, it is unlikely that, in general, non-thermal ionization/excitation by ${ }^{56} \mathrm{Ni}$ is the missing mechanism in our former approach (Dessart \& Hillier 2006a) since SNe events like $1999 \mathrm{br}$ or $2005 \mathrm{cs}$, which both boast a strong $\mathrm{H} \alpha$ line at the hydrogen recombination epoch, are also characterized by vanishingly small ${ }^{56} \mathrm{Ni}$ yields (Pastorello et al. 2004; Tsvetkov et al. 2006; Takáts \& Vinkó 2006; Pastorello et al. 2006). Furthermore, the $\mathrm{H} \alpha$ discrepancy appears at times when the photosphere still moves at $4000-5000 \mathrm{~km} \mathrm{~s}^{-1}$, velocities out to which ${ }^{56} \mathrm{Ni}$ is not expected to be mixed routinely (Fryxell et al. 1991; Kifonidis et al. 2000, 

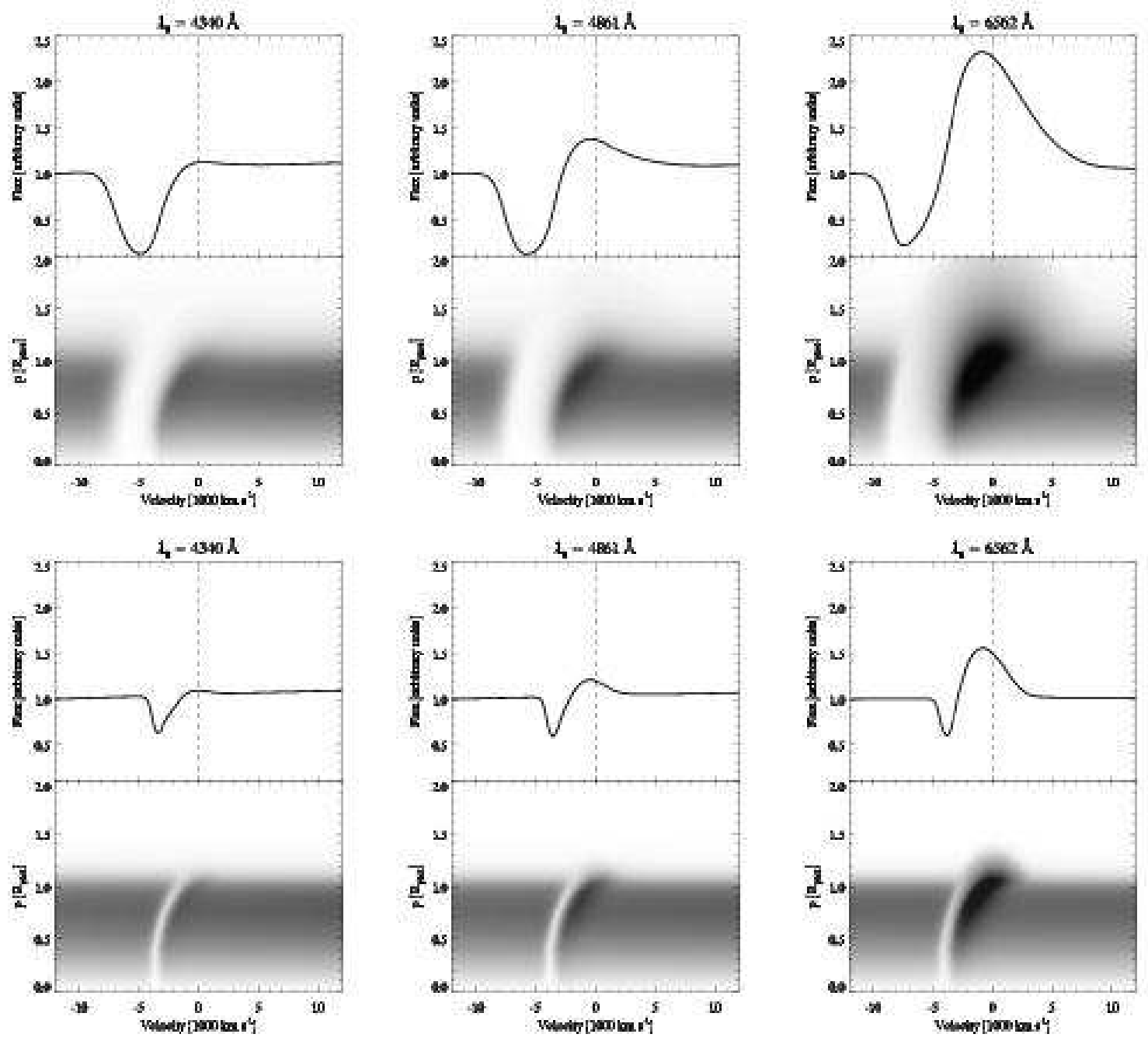

Figure 10. Grayscale images for $\mathrm{H} \gamma$ (left column), $\mathrm{H} \beta$ (middle column), and $\mathrm{H} \alpha$ (right column), of the quantity $p \cdot I(p)$ as a function of impact parameter $p$ and classical Doppler velocity $v=\left(\lambda / \lambda_{0}-1\right) c$, where $p$ is the impact parameter (in units of the photospheric radius $\mathrm{R}_{\mathrm{phot}}$ ), and $I(p)$ is the specific intensity along $p$ (at $v$ ). The panels in the top (bottom) row correspond to the time-dependent (steady-state) results obtained for the model A ejecta at 48.7 days after shock breakout. The top half of each panel gives the line profile flux, which, for each $v$, corresponds to sum of $p \cdot I(p)$ over all $p$ s, giving a vivid illustration of the $(p, v)$ sites contributing to the observed line profile. To emphasize the differences between lines and model assumptions, axis extrema are kept identical for all frames.

2003). The $\mathrm{H} \alpha$ problem in SN 1987A, for example, is already visible at just a few days after explosion, while Cobalt lines, testifying for the presence of radioactive material in the photosphere layers, were first seen at about 45 days after explosion (see, e.g., Pinto \& Woosley 1988), significantly later (but much earlier than anticipated by the theory at the time SN 1987A went off).

We start the comparison with optical and near-IR observations of SN 1999em on the 18th-19th of November (Leonard et al. 2002, Hamuy et al. 2001). These observations were modeled by Dessart \& Hillier (2006a) and we reproduce their Fig. 7 in the right panel of Fig. 15. In the left panel, we fit the observations with the synthetic spectrum computed for model A on day 35, and include the near-IR range to emphasize the concomitant strengthening of the Paschen lines in the time-dependent model. Timedependence effects are thus not limited to $\mathrm{H} \alpha$, nor to hydrogen Balmer lines exclusively, but operate as well on the Paschen series, and, even more generally, affect lines of all species. Note also that the predicted HeI line at $10830 \AA$ is too weak to fit the absorption feature at $1.03 \mu \mathrm{m}$, which, despite our neglect of CI contributions (Dessart \& Hillier 2006a), may require the additional con- 

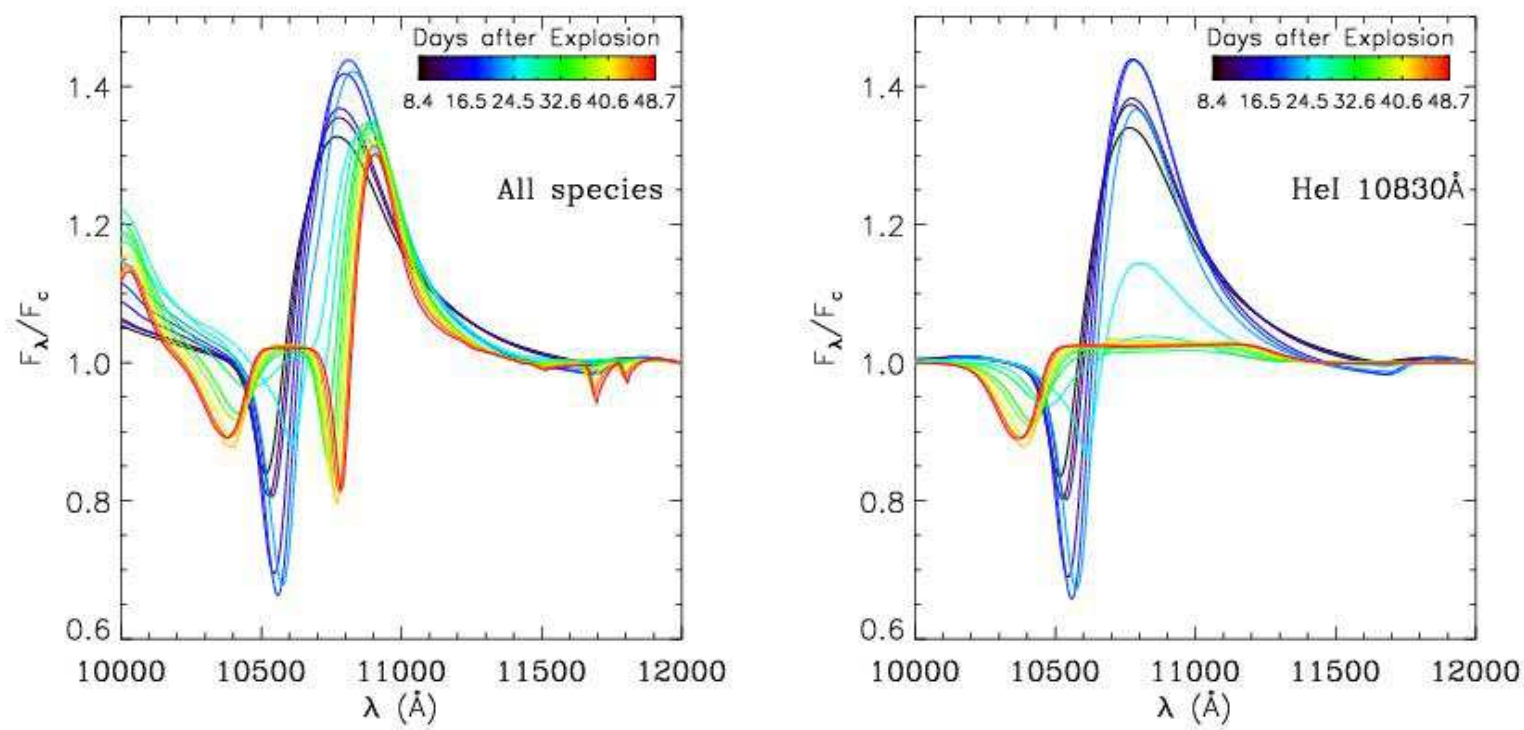

Figure 12. Left: Time sequence of the rectified synthetic flux over the $10000-12000 \AA$ range including all the bound-bound transitions. Main features are due to $\mathrm{P} \gamma$ and HeI $10830 \AA$. Right: Same as left, but this time, we only include HeI bound-bound transitions. Notice the flat-top and blueshifted absorption of HeI $10830 \AA$ at late times. [See the electronic edition of the Journal for a color version of this figure, and the text for discussion.]
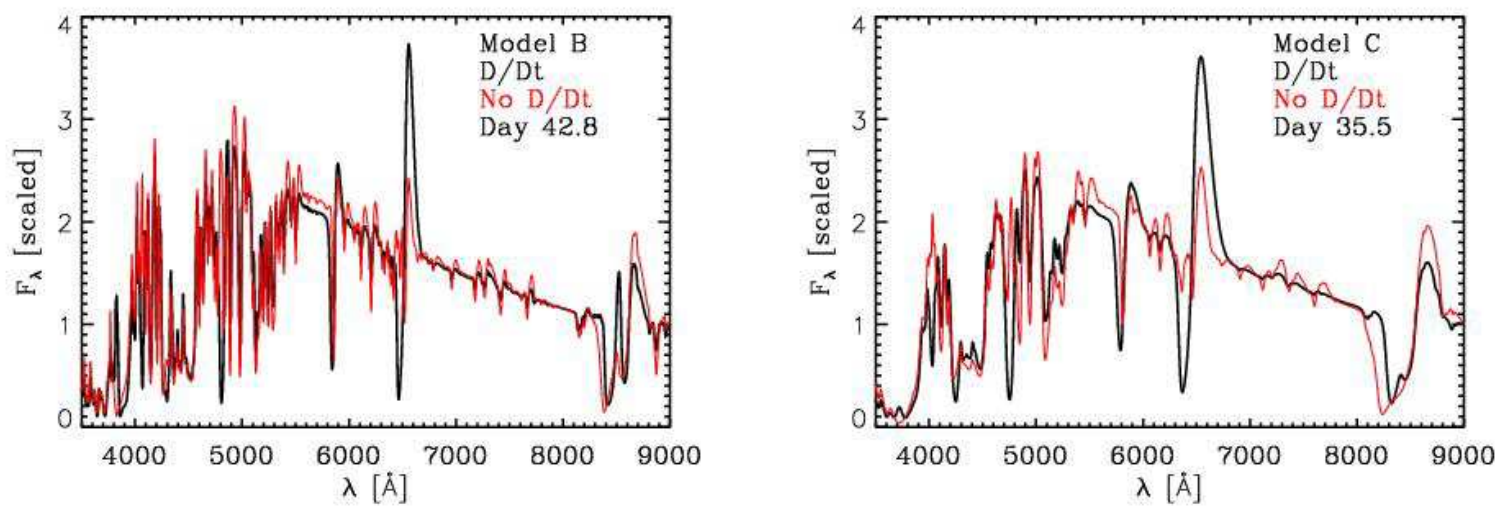

Figure 13. Optical synthetic spectra for the time-dependent (black) and steady-state (red) model B (left; day 42.8) and model C (right; day 35.5) at the last time in the sequence. [See the electronic edition of the Journal for a color version of this figure, and the text for discussion.]

tribution from the Cold Dense Shell at the ejecta/pre-SN-wind interface (Chugai et al. 2007). Note that the time-dependent model, unlike the steady-state model shown on the right, was not tuned to fit the observations. Hence, the adopted luminosity evolution may not be fully adequate for this event. An additional issue is that model A started at one week after explosion, while we demonstrated clearly in 3.2 the strong time-dependence effects occuring for HeI even at such early times, effects that will carry over to subsequent times and that may therefore be underestimated in these model sequences.

In Fig. 16 we compare synthetic spectra for Model A at 48.7 days after explosion, with allowance for time dependence (left panel) or assuming steady-state (right panel), with the observations of SN 1999em taken on the 15th of December 1999 (Leonard et al. 2002). The SED has changed significantly from that seen on the 19th of November 1999. There is more flux depletion in the UV, more metal line-blanketing in the optical, and $\mathrm{H} \alpha, \mathrm{NaI} \mathrm{D}$, and CaII $8500 \AA$ have strengthened. The steady-state model (right panel) reproduces well the overall shape of the SED, but what was a slight discrepancy in fitting the $\mathrm{H} \alpha$ line at earlier epochs now becomes a major discrepancy. By contrast, the time-dependent model reveals a strong and broad $\mathrm{H} \alpha$ line that closely matches the observed profile. The same agreement is reached for NaI D. A number of metal species are missing (scandium, barium) and the $\mathrm{CNO}$ abundances would also need adjustment (enhancement of carbon and oxygen abundances, and depletion of nitrogen; Dessart et al. 2007) but overall, the time-dependent model allows a much more satisfactory fit to observations and in particular $\mathrm{H} \alpha$, which is the defining feature of Type II SN spectra.

Note that the alternatives, an enhancement in turbulent velocity or flattening of the density distribution, do not improve the agreement with observations. The former has a weak or negligible effect at late times, while the latter leads to a large enhancement of the CaII $8500 \AA$ line strength, in stark contradiction with 

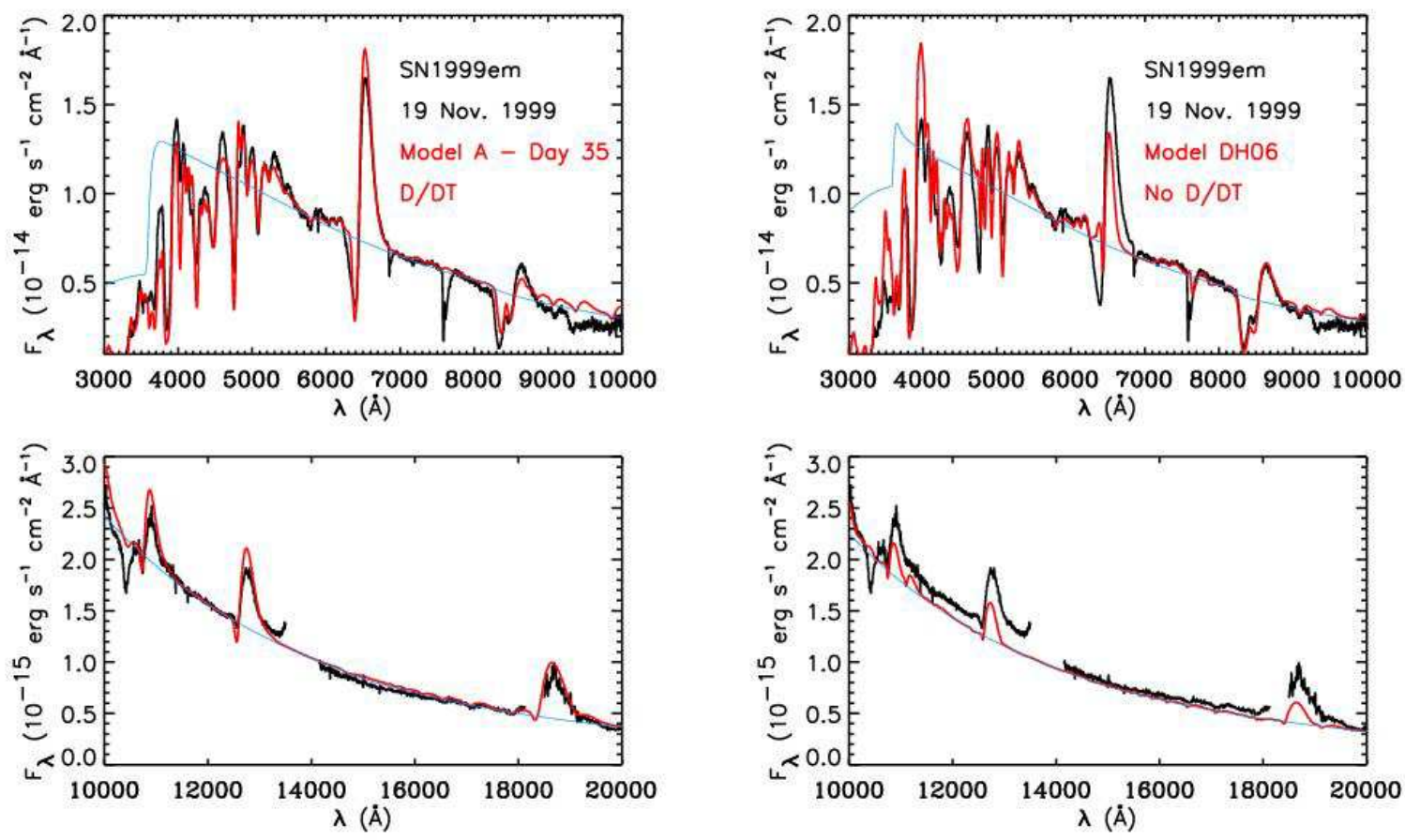

Figure 15. Left: Illustration of the match between observations (black) of SN 1999em on the 19th of November 1999 in the optical range (top; Leonard et al. 2002) and on the 18th of November 1999 in the near-IR range (bottom; Hamuy et al. 2001), with the reddened synthetic spectrum (red; blue: continuum-only) for the time-dependent model A at 35 days after explosion. We use the extinction law of Cardelli et al. (1988), with $E(B-V)=0.1$, and normalize the synthetic flux to the observed flux at $6100 \AA$, with a further $15 \%$ flux reduction in the near-IR (note the one day time difference between the two spectra). Right: Same as left, but this time using the equivalent steady-state model. [See the electronic edition of the Journal for a color version of this figure, and the text for discussion; see also \$3.2.7 and Fig. 7 in Dessart \& Hillier (2006a).]

observations. The key issue here is that one should not view the recombination rate as scaling with the density squared, but rather as the ion density times the free-electron density. Once the material has fully recombined, the absence of free-electrons and ions completely shrinks the recombination rate, and $\mathrm{H} \alpha$ becomes only weakly sensitive to modulations in the outer mass density distribution.

We finally show in Fig. 17 a comparison of Model B at 42.8 days after explosion, including time dependence (left panel) or assuming steady state (right panel), with the observations of SN 1999br on the 19th of May 1999 (Pastorello et al. 2004). The timedependence effects are similar to those described for SN 1999em on the 15th of December 1999, but they bear more strongly on the role of time dependence since, for the underluminous low-nickel-yield SN 1999br, non-thermal ionization/excitation is very unlikely to affect in any way $\mathrm{H} \alpha$ emission. Here again, time dependence effects lead to strong Balmer (and Paschen) lines, as observed, without recourse to extra ionizing/exciting sources or changes in the density profile. Furthermore, this shows that time-dependent effects are present in a wide variety of SN ejecta, even those characterized by a slow expansion rate.

\section{IMPLICATIONS}

\subsection{Abundance determinations}

The persistence of HeI $10830 \AA$ at 6 weeks after explosion in Model A suggests that the so-called detached absorption/emission lines are not exclusively associated with a stratification of abundances, with specific ions present at certain heights or mass shells in the ejecta. Here, the detached absorption/emission from HeI $10830 \AA$ stems purely from a time-dependence effect, and thus does not in principle require chemical stratification. Time-dependence effects can alter our interpretation of chemical stratification based on line profile measurements, e.g., velocity at maximum absorption. This is supported further by the time-dependence effects we see on the profile shape of strong lines, such as, for example, the hydrogen Balmer lines or NaI D. In the time-dependent model A at 48.7 days, we measure from the synthetic spectrum that included only the bound-bound transition of the relevant species a velocity at maximum absorption of $-7500 \mathrm{~km} \mathrm{~s}^{-1}$ for $\mathrm{H} \alpha,-5800 \mathrm{~km} \mathrm{~s}^{-1}$ for $\mathrm{H} \beta$, $-4900 \mathrm{~km} \mathrm{~s}^{-1}$ for $\mathrm{H} \gamma,-4000 \mathrm{~km} \mathrm{~s}^{-1}$ for NaI D (at 5890^), while in the same order, measurements performed on the synthetic spectrum for the steady-state model give $-3900 \mathrm{~km} \mathrm{~s}^{-1},-3500 \mathrm{~km} \mathrm{~s}^{-1}$, $-3400 \mathrm{~km} \mathrm{~s}^{-1}$, and $-3200 \mathrm{~km} \mathrm{~s}^{-1}$. Differences between these values are greater than those in photospheric velocity between the two models, which, respectively, are $4130 \mathrm{~km} \mathrm{~s}^{-1}$ and $3510 \mathrm{~km} \mathrm{~s}^{-1}$. Time-dependence effects operate in a non-linear fashion, affect- 

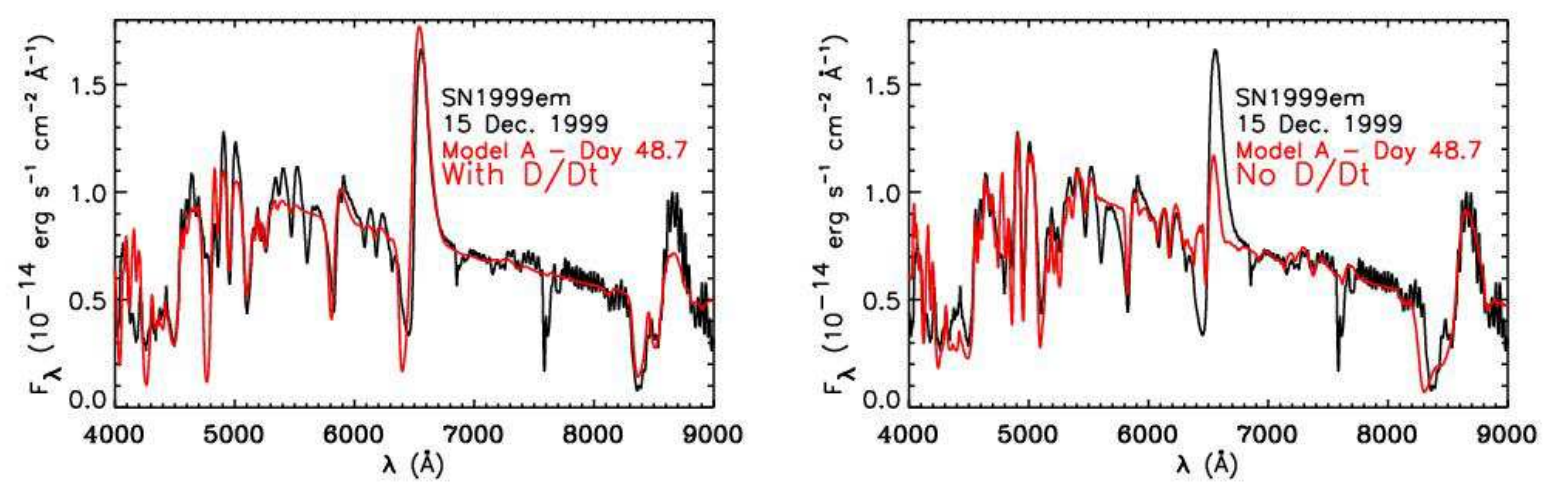

Figure 16. Same as Fig. 15 but for the observations of SN 1999em on the 15th of December 1999 (black; Leonard et al. 2002) and using the time-dependent model A at 48.7 days after explosion. [See the electronic edition of the Journal for a color version of this figure, and the text for discussion.]

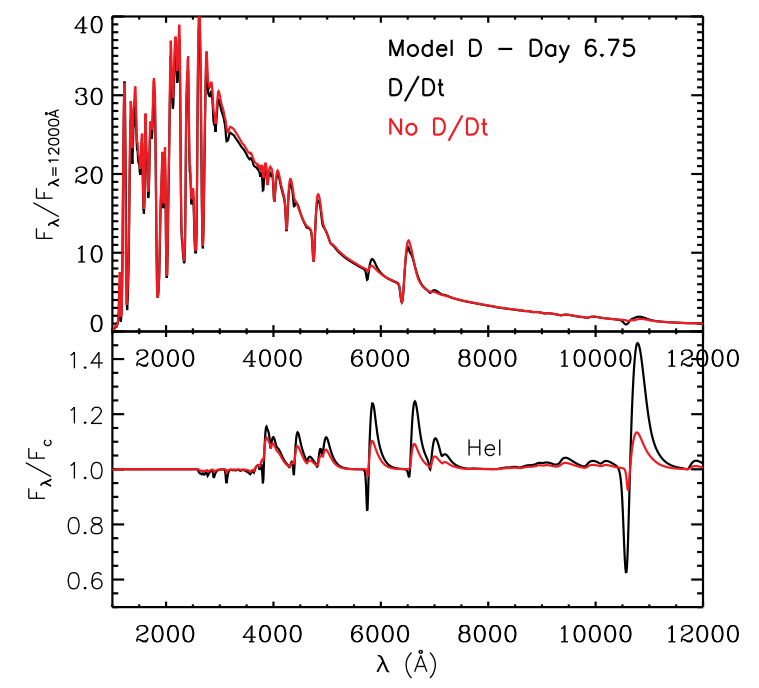

Figure 14. Top: Comparison of synthetic spectra obtained for model D at 6.8 days for the time-dependent (black) and steady-state (red) approaches. Bottom: Same as top, but for the synthetic rectified spectra accounting only for HeI bound-bound transitions. No time-dependent effect in the emergent light is seen for the other species treated in the calculation. (See text for discussion.)

ing lines and the continuum with a different magnitude, something to be expected since the continuum is mostly sensitive to the ionization state (a global quantity) while line profiles are affected by many parameters, such as the relevant ion level populations, the electron density, the radiation field etc. over a broad region in the SN ejecta.

Tomographic techniques, which aim at constructing the distribution of elements with mass in SN ejecta through the assumption of homologous expansion and kinematic measurements on (or fits to) line profiles (Stehle et al. 2005), may be compromised through the neglect, in the solution to the radiative transfer problem, of time-dependent terms in the statistical and radiative equilibrium equations. In the future, we will investigate such effects on inferences of chemical stratification in $\mathrm{SN}$ ejecta.

\subsection{Correction factors}

Type II SNe offer an attractive means to determine distances to galaxy hosts, and ultimately, constrain distances in the Universe. The so-called Expanding Photosphere Method (EPM; Kirshner \& Kwan 1974) has been used and improved upon to yield distances to a few nearby Type II SNe (Eastman \& Kirshner 1989; Schmidt et al. 1994; Eastman et al. 1996), although with a moderate level of success for SN $1999 \mathrm{em}$ for which the EPM distance (Hamuy et al. 2001; Leonard et al. 2002a; Elmhamdi et al. 2003) underestimates by $50 \%$ the Cepheid distance (Leonard et al. 2003) to the galaxy host. Using the updated correction factors of Dessart \& Hillier (2005b) partially cures this discrepancy, while even more consistent results require the use of tailored models, with their combined correction factors and color temperatures (Dessart \& Hillier 2006; Dessart et al. 2007).

Originally, the correction factors were introduced to account for the flux dilution that stems from the large electron-scattering optical depth contribution of the photosphere. However, as noted by Dessart \& Hillier (2005b), they also correct for the (strong) deviations from a blackbody distribution arising from the UV and optical line blanketing, particularly at later times.

Here, we investigate whether time-dependent models yield correction factors and associated color temperatures that depart systematically from steady-state models. Time dependence affects flux dilution by enhancing the electron density (and thus the magnitude of flux dilution) at all times, relative to a steady-state approach. However, it also affects the SED directly, both for the continuum (weakly) and for lines (strongly). We show in Fig. 18 the collection of correction factors versus color temperature for each set $B V$ (left), $B V I$ (middle), and $V I$ (right), for all models computed in the present study (51 models, shown as dots). For comparison, we overplot correction factors and associated color temperatures computed with steady-state CMFGEN models (37 in total, shown as crosses) for the study of SN 1999em (Dessart \& Hillier 2006a), as well as that for SNe 2005cs and 2006bp (Dessart et al. 2007).

At temperatures above $10,000 \mathrm{~K}$ we find no sizable systematic difference in correction factors computed with the time-dependent and steady-state approaches, which supports the use of the steadystate models of Type II SNe for distance determinations. At lower temperatures, particularly at temperatures less than $7000 \mathrm{~K}$, the new correction factors are somewhat smaller, and tend to lie at the lower edge of the distribution of correction factors calculated from steady-state models. They are also closer to those of Hamuy et al. 

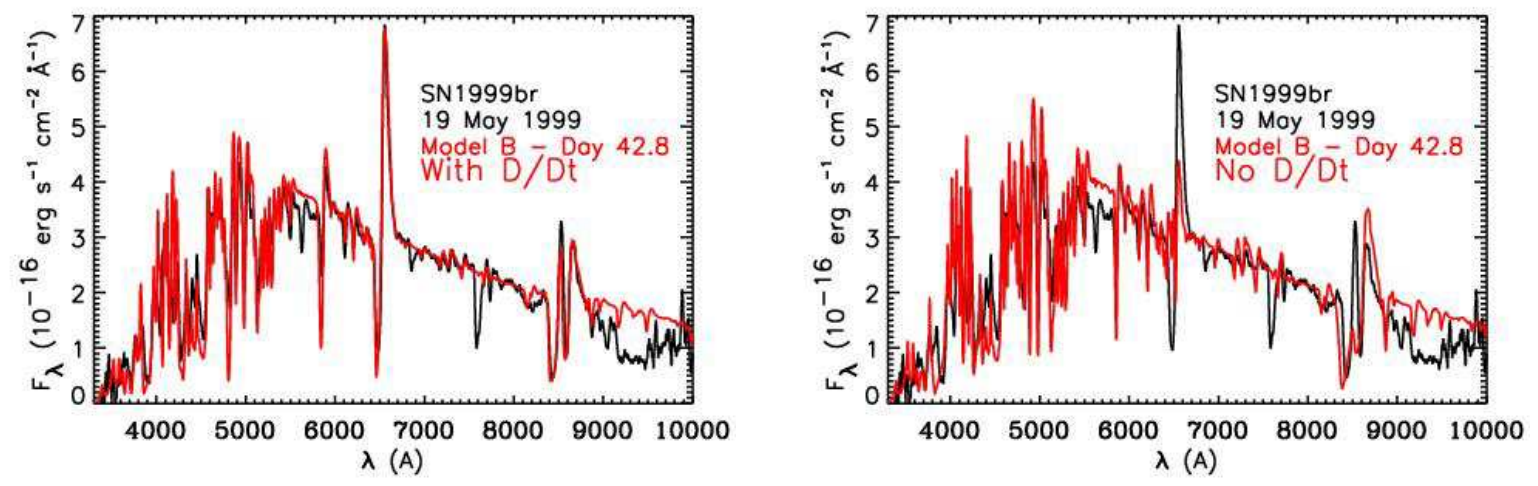

Figure 17. Same as Fig. 15 but for the observations of the underluminous event SN 1999br (black line; Pastorello et al. 2004) and using the time-dependent model B at 42.8 days after explosion. Synthetic spectra are reddened using the extinction law of Cardelli et al. (1988) with $E(B-V)=0.03$. [See the electronic edition of the Journal for a color version of this figure, and the text for discussion.]

(2001). The large scatter from model to model suggests that a tailored analysis is nonetheless preferred over the use of analytical correction factors. The departures we observe at low temperatures are not surprising - this is a regime where the time-dependent terms have a very large effect on spectral features, and a regime which we have tried to avoid in our EPM distance determinations. The inclusion of the time-dependent terms will allow us to better model the long-term evolution of SN spectra, and hence to extend the time baseline over which the EPM can be used. Further, the inclusion of these time-dependent terms is also important if we are to reduce systematic errors, and realize the fullest potential of the EPM technique.

\subsection{Polarization}

Through its effect on the ionization equilibrium of Type II SN ejecta, time dependence leads to an enhancement in the ejecta electron density. The ejecta optical depth falls off more rapidly than $1 / t^{2}$, but not as fast as it would for a steady-state configuration (Fig.6). Deeper layers should thus be revealed with a delay and the nebular phase be entered later in nature compared to steady-state predictions.

Moreover, the approximate linear scaling of the degree of polarization with the electron-scattering optical depth (Brown \& McLean 1977) suggests that, for a given degree of asymmetry, higher polarization measures will result from theoretical models generated assuming time-dependence, rather than stationarity. In Type II-P SNe, polarization is usually inferred to be at the $0.1 \%$ level during the plateau phase, and, although late time polarization measures are rare, $\mathrm{SN} 2004 \mathrm{dj}$ indicated that polarization can increase dramatically (here to $\sim 1 \%$ ) when the core is revealed (Leonard et al. 2006). Understanding what controls the ionization at such late times, in particular the respective roles of time dependence and radioactive decay from ${ }^{56} \mathrm{Co}$, is therefore critical for a proper interpretation of the degree of polarization from SN observations and the link to the asymmetry of the explosion.

\section{CONCLUSION}

We have presented an extension to the non-LTE model atmosphere code CMFGEN to treat time-dependent terms in the statistical and radiative equilibrium equations. We use implicit first order differencing, and the resulting equations are solved by a partial linearization technique in a manner virtually identical to that used to solve the steady state equations (Hillier \& Miller 1998). We confirm the findings of Utrobin \& Chugai (2005), which were related to $\mathrm{H} \alpha$ and BaII $6142 \AA$ at a few days after explosion in the spectrum of SN $1987 \mathrm{~A}$, that the time dependent terms are important for the analysis of Type II SN spectra.

We find that the inclusion of time-dependence terms in the statistical equilibrium equations produces ejecta, in Type II SN, that are systematically over-ionized relative to steady-state models. Spectroscopically, the associated changes in level populations and optical depths alter the line profiles of all species throughout the spectral range. Qualitatively, lines appear in general stronger and broader, as is seen for the Balmer, the Paschen, and the Brackett series of hydrogen or for NaI D. Exceptions (e.g. CaII $8500 \AA$ ) arise when the altered ionization inhibits the necessary recombination of a given ion (e.g., $\mathrm{Ca}^{++}$). Because of optical depth effects, the inclusion of time-dependent terms in the statistical equilibrium equations can even influence the ionization state of the gas in regions where the classic recombination times-scale is much shorter than the flow time-scale. Surprisingly, we predict the presence of HeI $10830 \AA 6$ weeks after the explosion, naturally caused by the frozen, and full, ionization of the outer ejecta. The resulting detached blueshifted absorption and flat-topped emission profile is supported by observations, although the magnitude of the absorption component may require the additional contribution from the cold dense shell that can form at the interface between the SN ejecta and the pre-SN wind (Chugai et al. 2007).

The effects of time dependence observed stem from the energy gain that follows changes in ionization and excitation, and from the similarity in recombination and expansion time-scales (crucial at large distances above the photosphere). The recombination timescale can be lengthened by optical-depth effects, and in some cases this may allow time-dependence effects to be important even at the photosphere. The present study indicates that neglecting time dependence may compromise the results of quantitative analyses of Type II SN spectra, affecting the inferred ejecta ionization (and its origin) or chemical abundances. In particular, time dependence offers a natural means to sustain strong and broad lines at late times, as observed in all Type II SN spectra, without invoking radioactive contributions. Such non-thermal excitation/ionization is expected to be relevant at the photosphere after a few months, but not as 

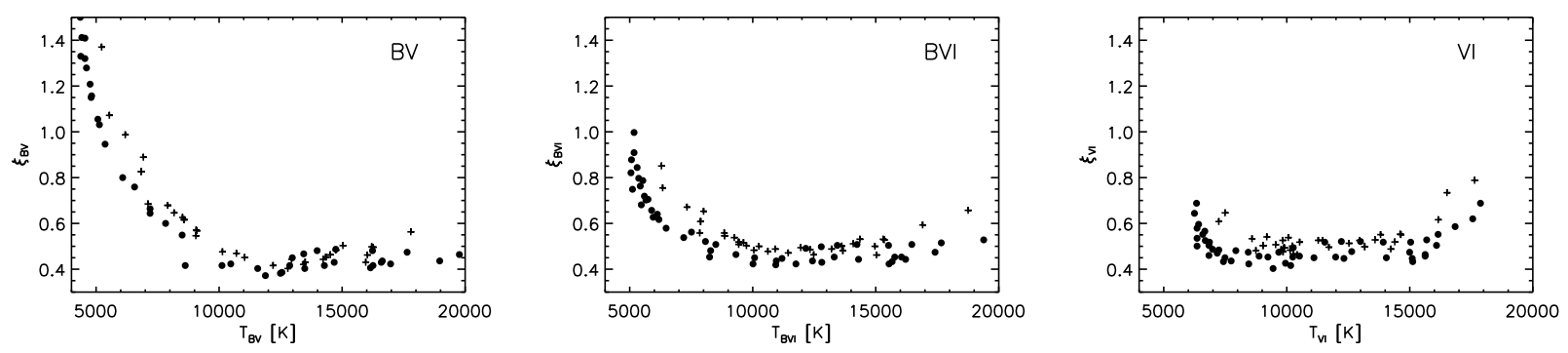

Figure 18. Correction factors $\xi_{S}$ versus corresponding color temperature $T_{S}$ for the 51 time-dependent models computed in the present study (dots), and shown for bandpass sets $B V$ (left), $B V I$ (middle), and $V I$ (right). For comparison, we also overplot crosses for the $\left\{\xi_{S}, T_{S}\right\}$ couples obtained with steadystate CMFGEN models for SN 1999em (Dessart \& Hillier 2006a), SNe 2005cs and 2006bp (Dessart et al. 2007). There is broad agreement between the time-dependent and steady-state predictions, although at low temperatures there is a tendency for the time-dependent correction factors to lie at the lower edge of the distribution for the steady-state models.

soon as a few weeks, and is subject to strong variations accross the SN class (SNe 1999 br or $2005 \mathrm{cs}$, which boast a strong $\mathrm{H} \alpha$ at the recombination epoch, have symptomatically low ${ }^{56} \mathrm{Ni}$ yields compared to the standard Type II-Plateau SN 1999em).

The time-dependence effects we observe just one week after explosion in the outer, fully ionized, SN ejecta also support the idea that the energy gain drawn out of recombining ions is not a fundamental driver. Time-dependence effects can lead to over-ionization for $\mathrm{He}, \mathrm{C}, \mathrm{N}, \mathrm{O}$, and metals, under fully-ionized hydrogen conditions. We thus surmise that time dependence should operate, with a magnitude to be determined, in $\mathrm{SN}$ ejecta of all types, primarily because they all combine the properties of fast expansion and low density.

We have investigated the potential impact of time dependence on the correction factors used in the Expanding Photosphere Method, or, essentially, whether the influence on the electron density generates a systematic shift in the magnitude of flux dilution. We find no sizable and systematic deviation from the correction factors obtained, at the same color temperature, with steady-state CMFGEN models. This supports our use of steady-state CMFGEN models for distance determinations based on early-time Type II SN observations (Dessart \& Hillier 2006a; Dessart et al. 2007). However, the good agreement at late times between time-dependent CMFGEN models and late time photospheric-phase observations motivates the extension of the time baseline to include the recombination epoch, thus allowing for multi-epoch observations that cover the entire Plateau phase, thereby reducing the errors on the inferred distance.

For the modeling of the longer evolution of Type II SN ejecta and their radiation, a time-dependent approach is warranted. To achieve a higher level of consistency, CMFGEN is under developement to follow as well the time-dependent evolution of the radiation field, together with options for chemical stratification and energy deposition from radioactive isotopes. This versatility will allow us to study $\mathrm{SN}$ ejecta of any type and over months after explosion.

\section{ACKNOWLEDGMENTS}

We thank Andrea Pastorello, Doug Leonard, and Mario Hamuy for providing optical spectra of SN 1999br and SN 1999em. L.D. acknowledges support for this work from the Scientific Discovery through Advanced Computing (SciDAC) program of the DOE, under grant numbers DE-FC02-01ER41184 and DE-FC0206ER41452, and from the NSF under grant number AST-0504947.
Table A1. Term comparison. Note that $\operatorname{PR}(\mathrm{n}=1) / \mathrm{RR}$ is the photoionization/recombination rate from/to level 1.

\begin{tabular}{|c|c|c|}
\hline \multirow{2}{*}{$\begin{array}{l}\text { Process } \\
\mathrm{R}\end{array}$} & \multicolumn{2}{|l|}{ Term } \\
\hline & $4.42 \times 10^{14}$ & $\mathrm{~cm}$ \\
\hline $\mathrm{v}$ & 7574 & $\mathrm{~km} \mathrm{~s}^{-1}$ \\
\hline $\mathrm{He}^{+}$density & $1.06 \times 10^{9}$ & $\mathrm{~cm}^{-3}$ \\
\hline $\mathrm{He}\left(1 \mathrm{~s}^{2}{ }^{1} \mathrm{~S}\right)$ density & $7.08 \times 10^{8}$ & $\mathrm{~cm}^{-3}$ \\
\hline Electron density & $9.92 \times 10^{9}$ & $\mathrm{~cm}^{-3}$ \\
\hline$t \exp$ & 6.75 & days \\
\hline$t_{\mathrm{rec}}(\mathrm{H})$ & 0.0045 & days \\
\hline $\mathrm{PR}(\mathrm{n}=1)$ & $1.8012 \times 10^{6}$ & $\mathrm{~cm}^{-3} \mathrm{~s}^{-1}$ \\
\hline RR-PR (n=1) & $8.0 \times 10^{2}$ & $\mathrm{~cm}^{-3} \mathrm{~s}^{-1}$ \\
\hline $2 \mathrm{p}^{1} \mathrm{P}^{o} \rightarrow 1 \mathrm{~s}^{2}{ }^{1} \mathrm{~S}$ & $2.15 \times 10^{3}$ & $\mathrm{~cm}^{-3} \mathrm{~s}^{-1}$ \\
\hline $2 \mathrm{p}^{3} \mathrm{P}^{o} \rightarrow 1 \mathrm{~s}^{2}{ }^{1} \mathrm{~S}$ & $4.49 \times 10^{2}$ & $\mathrm{~cm}^{-3} \mathrm{~s}^{-1}$ \\
\hline $2{ }^{1} S \rightarrow 1 s^{2}{ }^{1} S$ & $6.4 \times 10^{1}$ & $\mathrm{~cm}^{-3} \mathrm{~s}^{-1}$ \\
\hline$\sum_{n>2} n p^{1} \mathrm{P}^{o} \rightarrow 1 \mathrm{~s}^{2}{ }^{1} \mathrm{~S}$ & $3.00 \times 10^{2}$ & $\mathrm{~cm}^{-3} \mathrm{~s}^{-1}$ \\
\hline$\sum 2 \mathrm{~s}, 2 \mathrm{p}$ states $\rightarrow 1 \mathrm{~s}^{2}{ }^{1} \mathrm{~S}(\mathrm{col})$ & $3.62 \times 10^{2}$ & $\mathrm{~cm}^{-3} \mathrm{~s}^{-1}$ \\
\hline $\mathrm{Dn}(\mathrm{He}(\mathrm{n}=1)) / \mathrm{Dt}$ & $4.11 \times 10^{3}$ & $\mathrm{~cm}^{-3} \mathrm{~s}^{-1}$ \\
\hline
\end{tabular}

\section{APPENDIX A:}

We illustrate how the time-dependent terms can influence the He I ionization in SN models. We choose a model with a Hubble age of 6.75 days, and choose a single depth in the atmosphere for which helium is still appreciably ionized in the time-dependent model, but neutral when such terms are not included.

In Table A1 we list the various processes, and their rates, which influence the helium ionization balance. From this table we see the following: The most important process populating level $1 \mathrm{~s}^{2}$ (the ground state) is decays from the $2 \mathrm{p}^{1} \mathrm{P}^{o}$ state through the optically thick resonance transition, followed by the slight imbalance between photoionization from level $1 \mathrm{~s}^{2}$, and direct recombination to level $1 \mathrm{~s}^{2}$. Many other processes also play a role, including collisional de-excitation from the $2 \mathrm{~s} \& 2 \mathrm{p}$ levels, decay from the $2 \mathrm{p}^{3} \mathrm{P}^{o}$ state, and decays from other members of the $n p{ }^{1} \mathrm{P}^{o}(n>2)$ series. As expected, the sum of all these decay processes is the same as $\mathrm{Dn}(\mathrm{He}[\mathrm{n}=1]) / \mathrm{Dt}$. Note that $\mathrm{Dn}(\mathrm{He}(\mathrm{n}=1)) / \mathrm{Dt}=1.2 \times 10^{4} \mathrm{~cm}^{-3} \mathrm{~s}^{-1}$ if helium was to change from fully ionized, to fully neutral, over one day. Although not directly apparent from the table, most of the recombinations to excited states of neutral helium are balanced by ionizations from the $2 \mathrm{~s}$ and $2 \mathrm{p}$ states. 


\section{REFERENCES}

Baron, E., Nugent, P. E., Branch, D., Hauschildt, P. H., Turatto, M., \& Cappellaro, E. 2003, ApJ, 586, 1199

Brown, J. C., \& McLean, I. S. 1977, A\&A, 57, 141

Brown, P. J., et al. 2007, ApJ, 659, 1488

Chugai, N. N., Chevalier, R. A., \& Utrobin, V. P. 2007, ApJ, 662, 1136

Dessart, L., \& Hillier, D. J. 2005a, A\&A, 437, 667

Dessart, L., \& Hillier, D. J. 2005b, A\&A, 439, 671

Dessart, L., \& Hillier, D. J. 2006a, A\&A, 447, 691

Dessart, L., \& Hillier, J. 2006b, ArXiv Astrophysics e-prints, arXiv:astro-ph/0610136

Dessart et al. 2007, submitted to ApJ

Eastman, R. G., \& Kirshner, R. P. 1989, ApJ, 347, 771

Eastman, R. G., Schmidt, B. P., \& Kirshner, R. 1996, ApJ, 466, 911

Ensman, L., \& Burrows, A. 1992, ApJ, 393, 742

Fransson, C., \& Kozma, C. 1993, ApJ1, 408, L25

Fryxell, B., Arnett, D., \& Mueller, E. 1991, ApJ, 367, 619

Hamuy, M., et al. 2001, ApJ, 558, 615

Hamuy, M., et al. 2002, AJ, 124, 417

Hauschildt, P. H., 1992, ApJ, 398, 224

Hauschildt, P. H., Best, M., \& Wehrse, R. 1991, A\&A, 247, L21

Hillier, D. J. 1987, ApJs, 63, 965

Hillier, D. J. 1990, A\&A, 231, 116

Hillier, D. J., \& Miller, D. L. 1998, ApJ, 496, 407

Höflich, P. 1988, Proceedings of the Astronomical Society of Australia, 7, 434

Höflich, P. 2003, in Stellar Atmosphere Modeling, eds. Ivan Hubeny, Dimitri Mihalas, and Klaus Werner, Tuebingen, Germany, ASP Conference Proceedings, Vol. 288., p. 185

Jeffery, D.J. 1993 ApJ., 415, 734

Kasen, D., Thomas, R. C., \& Nugent, P. 2006, ApJ, 651, 366

Kifonidis, K., Plewa, T., Janka, H.-T., Mueller, E. 2000, ApJl, 531, L123

Kifonidis, K., Plewa, T., Janka, H.-T., Mueller, E. 2003, A\&A, 408, 621

Leonard, D. C., et al. 2002, PASP, 114, 35

Leonard, D. C., Kanbur, S. M., Ngeow, C. C., \& Tanvir, N. R. 2003, ApJ, 594, 247

Leonard, D. C., et al. 2006, Nature, 440, 505

Lucy, L. B. 1991, ApJ, 383, 308

Marion, G. H., Höflich, P., Vacca, W. D., \& Wheeler, J. C. 2003, ApJ, 591, 316

Mazzali, P. A., Lucy, L. B., \& Butler, K. 1992, A\&A, 258, 399

Mazzali, P. A., \& Lucy, L. B. 1998, MNRAS, 295, 428

Mihalas, D. 1978, San Francisco, W. H. Freeman and Co..

Mitchell, R. C., Baron, E., Branch, D., Lundqvist, P., Blinnikov, S., Hauschildt, P. H., \& Pun, C. S. J. 2001, ApJ, 556, 979

Osterbrock, D. E. 1974, Astrophysics of gaseous nebulae, W. H. Freeman and Co.

Pastorello, A., et al. 2004, MNRAS, 347, 74

Pastorello, A., et al. 2006, MNRAS, 370, 1752 (P06)

Pinto, P. A., \& Woosley, S. E. 1988, Nature, 333, 534

Pinto, P. A., \& Eastman, R. G. 2000, ApJ, 530, 744

Pinto, P. A., \& Eastman, R. G. 2000, ApJ, 530, 757

Quimby, R. M., Wheeler, J. C., Höflich, P., Akerlof, C. W., Brown, P. J., \& Rykoff, E. S. 2007, ArXiv e-prints, 705, arXiv:0705.3478 Schmutz, W., Abbott, D. C., Russell, R. S., Hamann, W.-R., \& Wessolowski, U. 1990, ApJ, 355, 255

Smartt, S. J., Gilmore, G. F., Tout, C. A., \& Hodgkin, S. T. 2002,
ApJ, 565, 1089

Spyromilio, J., Stathakis, R. A., Cannon, R. D., Waterman, L., Couch, W. J., \& Dopita, M. A. 1991, MNRAS, 248, 465

Stehle, M., Mazzali, P. A., Benetti, S., \& Hillebrandt, W. 2005, MNRAS, 360, 1231

Takáts, K., \& Vinkó, J. 2006, MNRAS, 372, 1735

Tsvetkov, D. Y., Volnova, A. A., Shulga, A. P., Korotkiy, S. A., Elmhamdi, A., Danziger, I. J., \& Ereshko, M. V. 2006, A\&A, 460, 769

Utrobin, V. P., \& Chugai, N. N. 2005, A\&A, 441, 271 